\title{
n-Hosoya polynomials for Pentagonal Chains
}

Ali Aziz Ali

aliazizali1933@yahoo.com

Academic

University of Mosul

Received on: 02/05/2011

\section{Hadeel Abdullah Meshw}

\section{Directorate of General Education} Nineveh

Accepted on: 21/06/2011

\begin{abstract}
The diameter, with respect to the $\mathrm{n}$-distance of the graph $\mathrm{PC}_{\mathrm{m}}$ which represents a straight chain consisting of $\mathrm{m}$ pentagonal rings, is obtained in this paper. The $\mathrm{n}$ Hosoya polynomial of $\mathrm{PC}_{\mathrm{m}}$, for all $\mathrm{m}$ and $\mathrm{n}$, where $3 \leq \mathrm{n} \leq 3 \mathrm{~m}+2$, is also obtained.

Keywords: n-distance , n-Hosoya polynomials, Pentagonal Chains

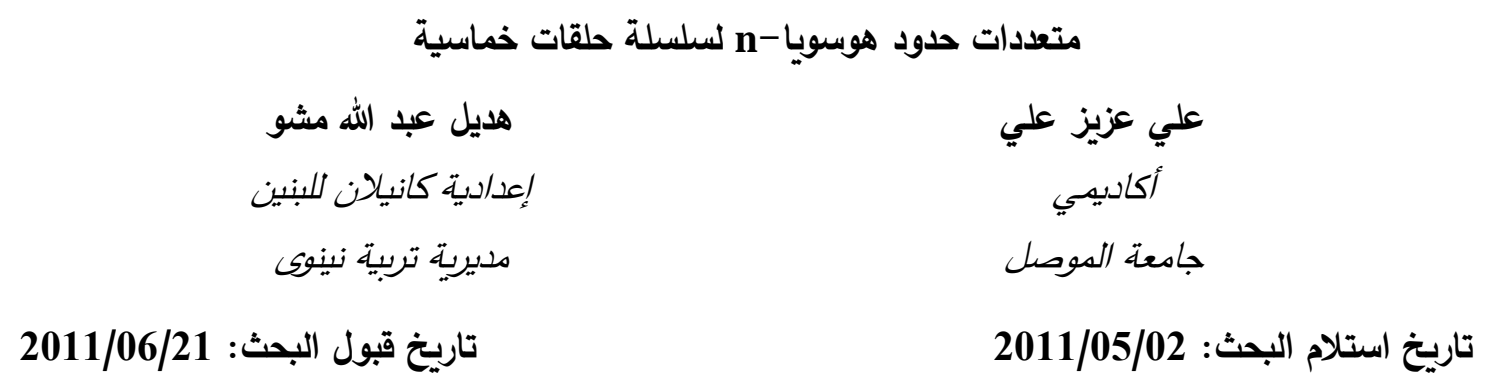

الملخص

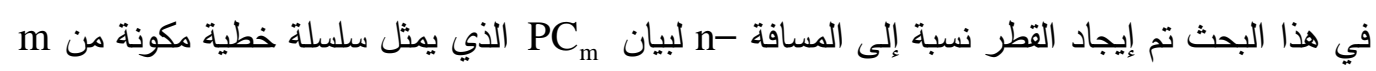

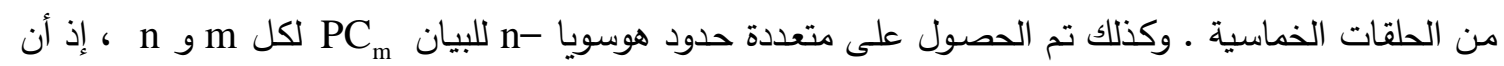
$.3 \leq \mathrm{n} \leq 3 \mathrm{~m}+2$

الكلمات المفتاحية: المسافة-n ، 3 ، متعددة حدود هوسويا-n ، سلاسل خماسية.

1

نتبع المفاهيم والمصطلحات في بحثنا هذا كما وردت وعرفت في المصادر [5,6,7]. ليكن v رأسا في

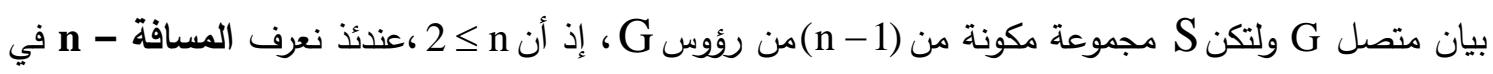

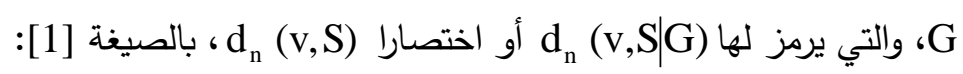

$\mathrm{d}_{\mathrm{n}}(\mathrm{v}, \mathrm{S})=\min \{\mathrm{d}(\mathrm{v}, \mathrm{u}): \mathrm{u} \in \mathrm{S}\}$

يعرف القطر - n مالذي يرمز له

$\operatorname{diam}_{\mathrm{n}} \mathrm{G}=\max \left\{\mathrm{d}_{\mathrm{n}}(\mathrm{v}, \mathrm{S}): \mathrm{v} \in \mathrm{V}, \mathrm{S} \subseteq \mathrm{V}(\mathrm{G}),|\mathrm{S}|=\mathrm{n}-1\right\}$

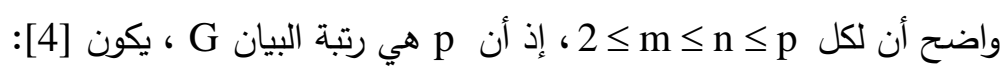

$\operatorname{diam}_{\mathrm{n}} \mathrm{G} \leq \operatorname{diam}_{\mathrm{m}} \mathrm{G} \leq \operatorname{diam} \mathrm{G}$


دليل وينر - n للبيان G ، والذي يرمز له بـ (G) يعرف كالآتي [1]:

$\mathrm{W}_{\mathrm{n}}(\mathrm{G})=\sum_{\substack{(\mathrm{V}, \mathrm{S}) \\ \mathrm{v} \in \mathrm{V}, S \subseteq \mathrm{V}}} \mathrm{d}_{\mathrm{n}}(\mathrm{v}, \mathrm{S})$

حيث يؤخذ المجموع على جميع الأزواج المرتبة (v,S)، لكل veV(G)، ولكل . $|\mathrm{S}|=\mathrm{n}-1$

تعرف متعددة حدود هوسويا-n للبيان المتصل G) والتي يرمز لها H (G; $\mathrm{H}_{\mathrm{n}}(\mathrm{G} ; \mathrm{x})=\sum_{(\mathrm{v}, \mathrm{S})} \mathrm{x}^{\mathrm{d}_{\mathrm{n}}(\mathrm{v}, \mathrm{S})}$,

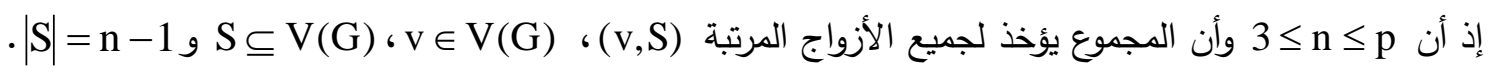
ويمكن التعبير عن Hin

$\mathrm{H}_{\mathrm{n}}(\mathrm{G} ; \mathrm{x})=\sum_{\mathrm{k}=0}^{\delta_{\mathrm{n}}} \mathrm{C}_{\mathrm{n}}(\mathrm{G}, \mathrm{k}) \mathrm{x}^{\mathrm{k}}$,

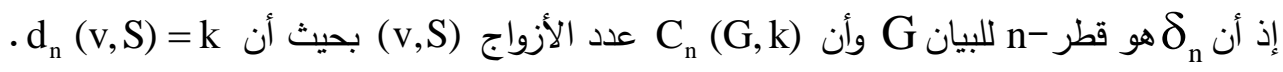
مما تقدم نستنتج أن:

$\mathrm{W}_{\mathrm{n}}(\mathrm{G})=\sum_{\mathrm{k}=1}^{\delta_{\mathrm{n}}} \mathrm{k} \mathrm{C}_{\mathrm{n}}(\mathrm{G}, \mathrm{k})=\left.\frac{\mathrm{d}}{\mathrm{dx}} \mathrm{H}_{\mathrm{n}}(\mathrm{G} ; \mathrm{x})\right|_{\mathrm{x}=1}$.

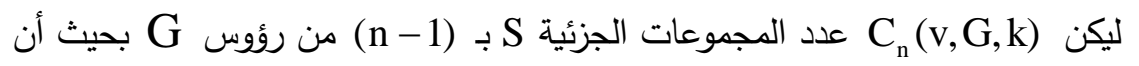

$\mathrm{d}_{\mathrm{n}}(\mathrm{v}, \mathrm{S})=\mathrm{k}, \mathrm{n} \geq 3,0 \leq \mathrm{k} \leq \delta_{\mathrm{n}}$,

عندئذ نعرف متعدة حدود هوسويا-n للرأس v في والتي يرمز لها H $\mathrm{H}_{\mathrm{n}}(\mathrm{v}, \mathrm{G} ; \mathrm{x})=\sum_{\mathrm{k} \geq 0} \mathrm{C}_{\mathrm{n}}(\mathrm{v}, \mathrm{G}, \mathrm{k}) \mathrm{x}^{\mathrm{k}}$.

$\mathrm{H}_{\mathrm{n}}(\mathrm{G} ; \mathrm{x})=\sum_{\mathrm{v} \in \mathrm{V}} \mathrm{H}_{\mathrm{n}}(\mathrm{v}, \mathrm{G} ; \mathrm{x})$

ملاحظة : إذا كانت A مجموعة جزئية غير خالية من V(G) فأننا نعرف [4]: $\mathrm{H}_{\mathrm{n}}(\mathrm{A}, \mathrm{G} ; \mathrm{x})=\sum_{\mathrm{a} \in \mathrm{A}} \mathrm{H}_{\mathrm{n}}(\mathrm{a}, \mathrm{G} ; \mathrm{x})$. وإذا كانت

$\mathrm{H}_{\mathrm{n}}(\mathrm{G} ; \mathrm{x})=\sum_{\mathrm{i}=1}^{\mathrm{r}} \mathrm{H}_{\mathrm{n}}\left(\mathrm{A}_{\mathrm{i}}, \mathrm{G} ; \mathrm{x}\right)$.

في هذا البحث نجد صيغة لحساب قطر - للبيان PC الموضح في الثكل 2.1، ومن ثم نجد

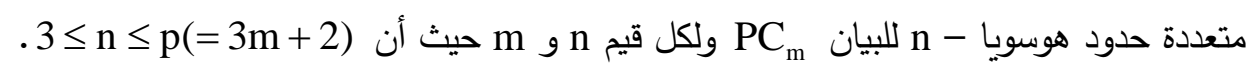

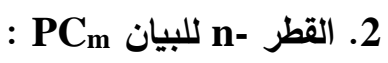


يُعرف البيان PC على أنه مكون من m من الحلقات الخماسية المتتابعة إذ أن كل حلقتين متتابعتين

تثتركان بحافة واحدة فقط مكونةً سلسلة خطية كما هو في الثكل 2.1 .
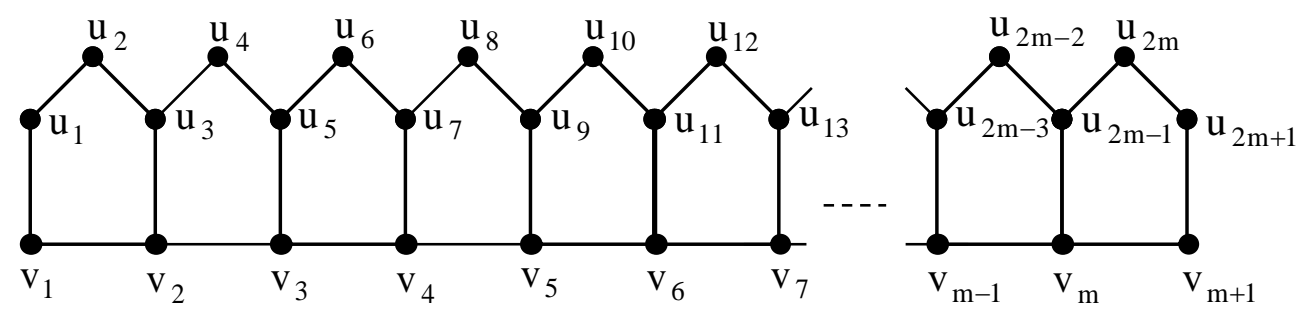

PC الثكل 2.1 - البيان

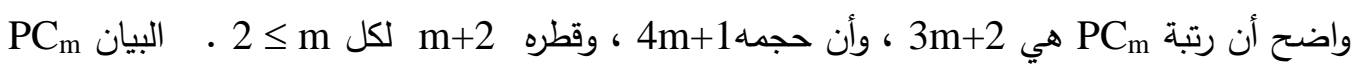

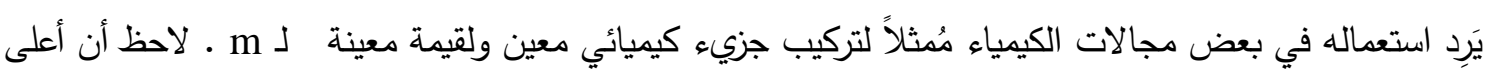

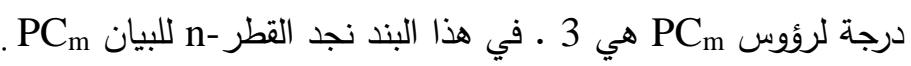

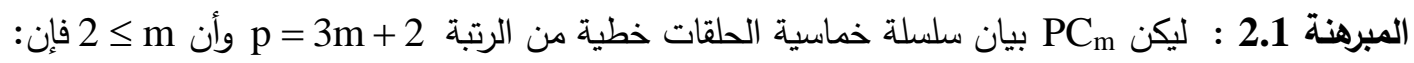

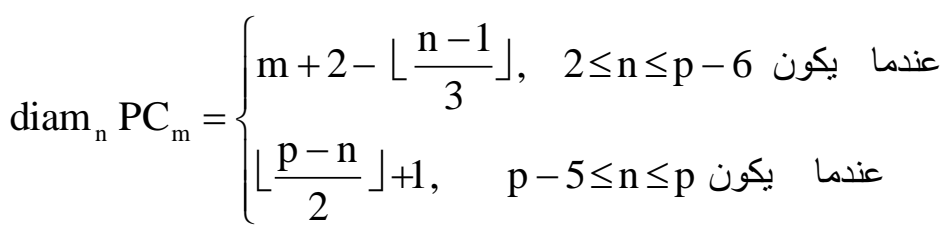

البرهان: من الشكل 2.1 ، نلاحظ أن:

$\operatorname{diam}_{\mathrm{n}} \mathrm{PC}_{\mathrm{m}}=\mathrm{e}_{\mathrm{n}}\left(\mathrm{u}_{1}\right)$.

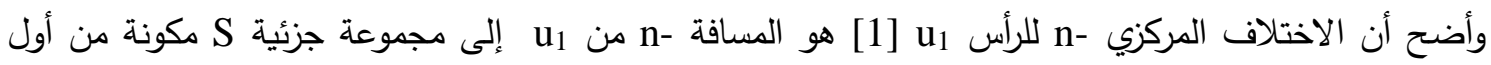

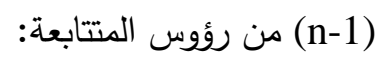

$\mathrm{u}_{2 \mathrm{~m}}, \mathrm{u}_{2 \mathrm{~m}+1} ; \mathrm{u}_{2 \mathrm{~m}-2}, \mathrm{u}_{2 \mathrm{~m}-1}, \mathrm{v}_{\mathrm{m}+1} ; \mathrm{u}_{2 \mathrm{~m}-4}, \mathrm{u}_{2 \mathrm{~m}-3}, \mathrm{v}_{\mathrm{m}} ; \ldots ; \mathrm{u}_{2 \mathrm{~m}-2 \mathrm{j}}, \mathrm{u}_{2 \mathrm{~m}-2 \mathrm{j}+1}, \mathrm{v}_{\mathrm{m}-\mathrm{j}+2} ; \ldots ; \mathrm{u}_{6}$ $, \mathrm{u}_{7}, \mathrm{v}_{5} ; \mathrm{u}_{5}, \mathrm{v}_{4} ; \mathrm{u}_{4}, \mathrm{v}_{3} ; \mathrm{u}_{3}, \mathrm{v}_{2} ; \mathrm{u}_{2}, \mathrm{v}_{1}$.

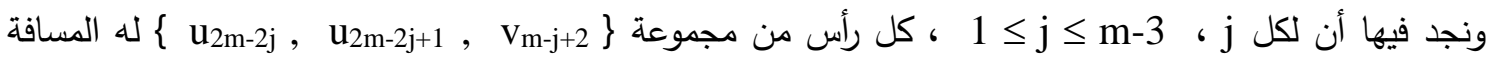

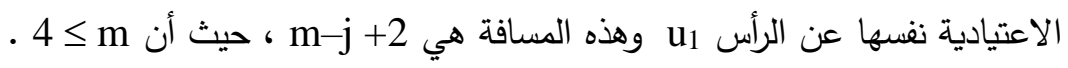

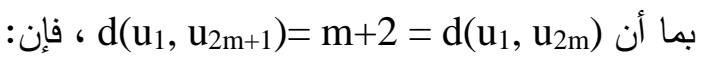

$\mathrm{e}_{\mathrm{n}}\left(\mathrm{u}_{1}\right)=\mathrm{m}+2-\left\lfloor\frac{\mathrm{n}-1}{3}\right\rfloor, \forall 2 \leq \mathrm{n} \leq \mathrm{p}-6$

فضلاً عن ذلك نجد أن:

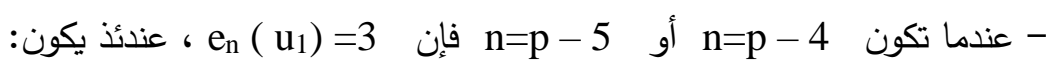
$\mathrm{e}_{\mathrm{n}}\left(\mathrm{u}_{1}\right)=\mathrm{d}_{\mathrm{n}}\left(\mathrm{u}_{1}, \mathrm{~S}\right), \mathrm{S} \subseteq \mathrm{V}\left(\mathrm{PC}_{\mathrm{m}}\right)-\left\{\mathrm{u}_{1}, \mathrm{u}_{2}, \mathrm{u}_{3}, \mathrm{v}_{1}, \mathrm{v}_{2}\right\},|\mathrm{S}|=\mathrm{p}-5$ or $\mathrm{p}-6$

- وعندما يكون $\mathrm{e}_{\mathrm{n}}\left(\mathrm{u}_{1}\right)=\mathrm{d}_{\mathrm{n}}\left(\mathrm{u}_{1}, \mathrm{~S}\right), \mathrm{S} \subseteq \mathrm{V}\left(\mathrm{PC}_{\mathrm{m}}\right)-\left\{\mathrm{u}_{1}, \mathrm{u}_{2}, \mathrm{v}_{1}\right\},|\mathrm{S}|=\mathrm{p}-3$ or $\mathrm{p}-4$

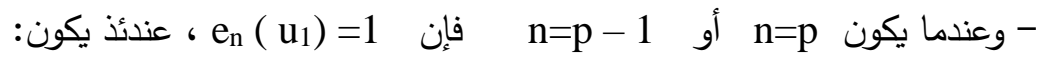
$\mathrm{e}_{\mathrm{n}}\left(\mathrm{u}_{1}\right)=\mathrm{d}_{\mathrm{n}}\left(\mathrm{u}_{1}, \mathrm{~S}\right), \mathrm{S} \subseteq \mathrm{V}\left(\mathrm{PC}_{\mathrm{m}}\right)-\left\{\mathrm{u}_{1}\right\},|\mathrm{S}|=\mathrm{p}-1$ or $\mathrm{p}-2$ 
a وبها يتم البرهان.

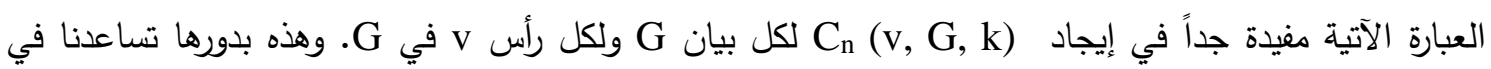

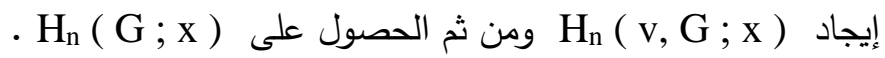

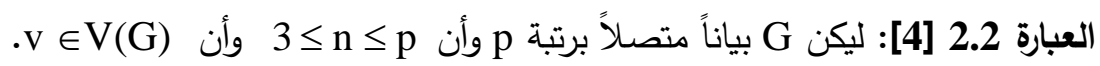

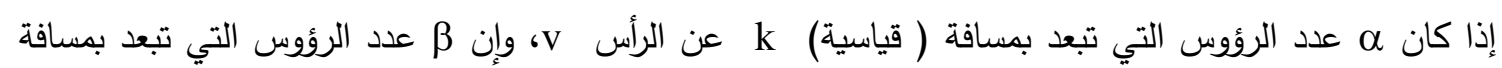
$C_{n}(v, G, k)=\left(\begin{array}{c}\alpha+\beta \\ n-1\end{array}\right)-\left(\begin{array}{c}\beta \\ n-1\end{array}\right)$

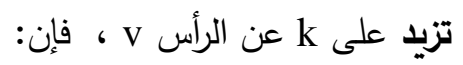

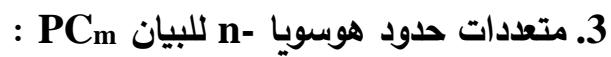

$$
\mathrm{H}_{\mathrm{n}}\left(\mathrm{PC}_{\mathrm{m}} ; \mathrm{x}\right)=\sum_{\mathrm{k}=0}^{\delta_{\mathrm{n}}} \mathrm{C}_{\mathrm{n}}\left(\mathrm{PC}_{\mathrm{m}}, \mathrm{k}\right) \mathrm{x}^{\mathrm{k}}
$$

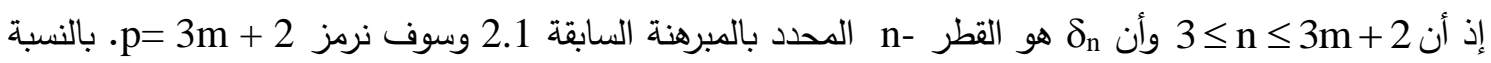

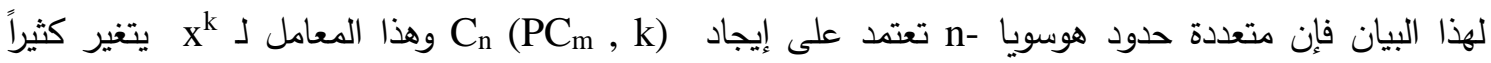
بالنسبة إلى قيمة k ولذلك نحتاج إلى معالجة حالات متعددة . وسوف نجزء $\mathrm{V}=\left\{\mathrm{v}_{1}, \mathrm{v}_{2}, \ldots, \mathrm{v}_{\mathrm{m}+1}\right\}, \mathrm{U}=\left\{\mathrm{u}_{1}, \mathrm{u}_{2}, \ldots, \mathrm{u}_{2 \mathrm{~m}}, \mathrm{u}_{2 \mathrm{~m}+1}\right\}$

رئيستين كالآتي ( لاحظ الثكل 2.1 ):

$$
\begin{aligned}
& \mathrm{C}_{\mathrm{n}}\left(\mathrm{V}, \mathrm{PC}_{\mathrm{m}}, \mathrm{k}\right)=\sum_{\mathrm{i}=1}^{\mathrm{m}+1} \mathrm{C}_{\mathrm{n}}\left(\mathrm{v}_{\mathrm{i}}, \mathrm{PC}_{\mathrm{m}}, \mathrm{k}\right) \\
& \text { والآن نعرف لكل : } 2 \text { : } \\
& \mathrm{C}_{\mathrm{n}}\left(\mathrm{U}, \mathrm{PC}_{\mathrm{m}}, \mathrm{k}\right)=\sum_{\mathrm{i}=1}^{2 \mathrm{~m}+1} \mathrm{C}_{\mathrm{n}}\left(\mathrm{u}_{\mathrm{i}}, \mathrm{PC}_{\mathrm{m}}, \mathrm{k}\right) \\
& \mathrm{C}_{\mathrm{n}}\left(\mathrm{PC}_{\mathrm{m}}, \mathrm{k}\right)=\mathrm{C}_{\mathrm{n}}\left(\mathrm{V}, \mathrm{PC}_{\mathrm{m}}, \mathrm{k}\right)+\mathrm{C}_{\mathrm{n}}\left(\mathrm{U}, \mathrm{PC}_{\mathrm{m}}, \mathrm{k}\right)
\end{aligned}
$$$$
\text { واضح أن: }
$$

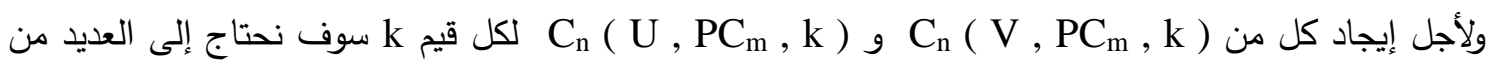$$
\text { العبارات والتي سوف نبرهنها فيما يأتي. }
$$

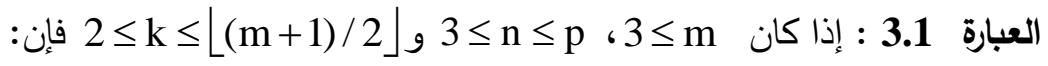$$
\mathrm{C}_{\mathrm{n}}\left(\mathrm{V}, \mathrm{PC}_{\mathrm{m}}, \mathrm{k}\right)=2\left(\begin{array}{c}
\mathrm{p}-3 \mathrm{k}+3 \\
\mathrm{n}-1
\end{array}\right)+(\mathrm{m}-2 \mathrm{k}+3)\left(\begin{array}{c}
\mathrm{p}-6 \mathrm{k}+8 \\
\mathrm{n}-1
\end{array}\right)-2\left(\begin{array}{c}
\mathrm{p}-6 \mathrm{k}+3 \\
\mathrm{n}-1
\end{array}\right)-2\left(\begin{array}{c}
\mathrm{p}-6 \mathrm{k}+6 \\
\mathrm{n}-1
\end{array}\right)
$$

$$
-(\mathrm{m}-2 \mathrm{k}+1)\left(\begin{array}{c}
\mathrm{p}-6 \mathrm{k}+2 \\
\mathrm{n}-1
\end{array}\right)
$$

البرهان: نلاحظ وجود ثلاثة حالات لـ V ، $\mathrm{i} \in\{1,2, \ldots, \mathrm{k}-1 ; \mathrm{m}-\mathrm{k}+3, \mathrm{~m}-\mathrm{k}+4, \ldots, \mathrm{m}+1\}$ الحالة (1): عندما تكون 
لأجل

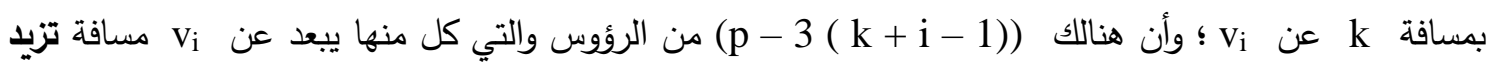
على k. لذلك باستعمال العبارة 2.2 نحصل على عنى:

$\mathrm{C}_{\mathrm{n}}\left(\mathrm{v}_{\mathrm{i}}, \mathrm{PC}_{\mathrm{m}}, \mathrm{k}\right)=\left(\begin{array}{c}\mathrm{p}-3(\mathrm{k}+\mathrm{i}-2) \\ \mathrm{n}-1\end{array}\right)-\left(\begin{array}{c}\mathrm{p}-3(\mathrm{k}+\mathrm{i}-1) \\ \mathrm{n}-1\end{array}\right)$.

بالنسبة لدالة المسافة فإن البيان PC يمتلك نوعاً من التماثل عندما نبدأ من V1 ونفسها إذا بدأنا برأس الانتهاء

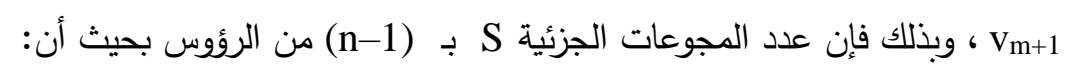
$\mathrm{d}_{\mathrm{n}}\left(\mathrm{v}_{\mathrm{i}}, \mathrm{S}\right)=\mathrm{k}, \mathrm{m}-\mathrm{k}+3 \leq \mathrm{i} \leq \mathrm{m}+1$

هو المعطى نفسه في (3.2) وعليه فإن العدد الكلي للأزواج (Vi , S) في هذه الحالة هو:

$2 \sum_{i=1}^{k-1}\left[\left(\begin{array}{c}p-3(k+i-2) \\ n-1\end{array}\right)-\left(\begin{array}{c}p-3(k+i-1) \\ n-1\end{array}\right)\right]=2\left[\left(\begin{array}{c}p-3 k+3 \\ n-1\end{array}\right)-\left(\begin{array}{c}p-6 k+6 \\ n-1\end{array}\right)\right]$

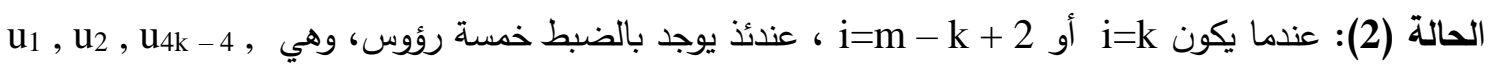
uluk - 3, v2k على k من الرأس Vk • م وعليه، فإن:

$\mathrm{C}_{\mathrm{n}}\left(\mathrm{v}_{\mathrm{k}}, \mathrm{PC}_{\mathrm{m}}, \mathrm{k}\right)=\left(\begin{array}{c}\mathrm{p}-6 \mathrm{k}+8 \\ \mathrm{n}-1\end{array}\right)-\left(\begin{array}{c}\mathrm{p}-6 \mathrm{k}+3 \\ \mathrm{n}-1\end{array}\right)$.

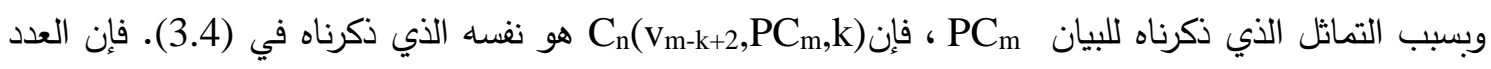

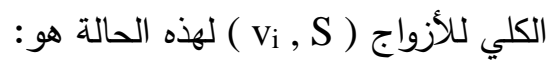

$2\left(\begin{array}{c}\mathrm{p}-6 \mathrm{k}+8 \\ \mathrm{n}-1\end{array}\right)-2\left(\begin{array}{c}\mathrm{p}-6 \mathrm{k}+3 \\ \mathrm{n}-1\end{array}\right)$

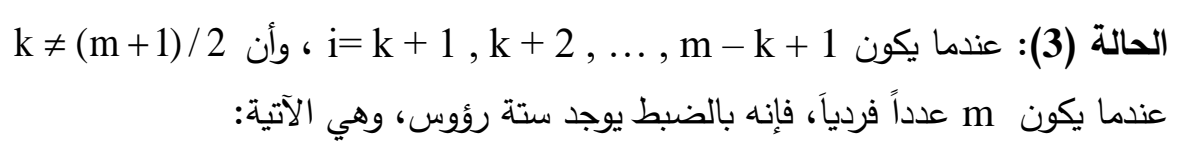

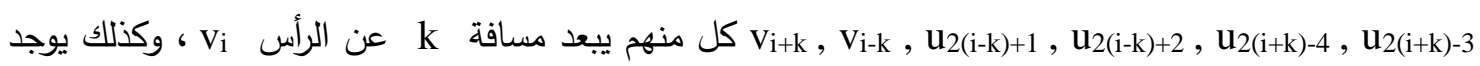

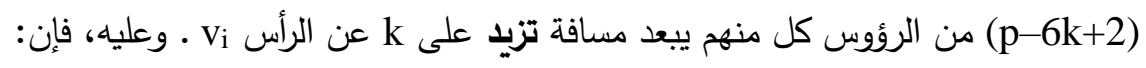

$\mathrm{C}_{\mathrm{n}}\left(\mathrm{v}_{\mathrm{i}}, \mathrm{PC}_{\mathrm{m}} ; \mathrm{k}\right)=\left(\begin{array}{c}\mathrm{p}-6 \mathrm{k}+8 \\ \mathrm{n}-1\end{array}\right)-\left(\begin{array}{c}\mathrm{p}-6 \mathrm{k}+2 \\ \mathrm{n}-1\end{array}\right)$

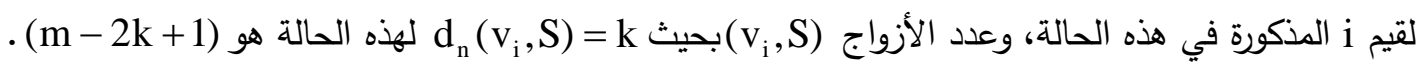

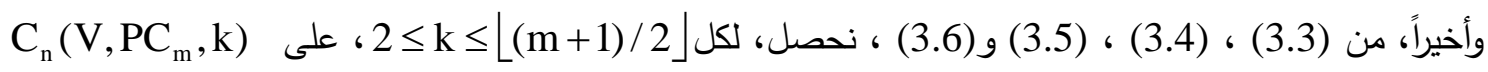

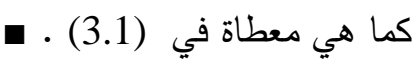

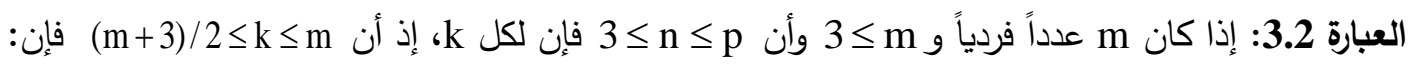

$\mathrm{C}_{\mathrm{n}}\left(\mathrm{V}, \mathrm{PC}_{\mathrm{m}}, \mathrm{k}\right)=2\left(\begin{array}{c}\mathrm{p}-3 \mathrm{k}+3 \\ \mathrm{n}-1\end{array}\right)$ 
البرهان: لأجل

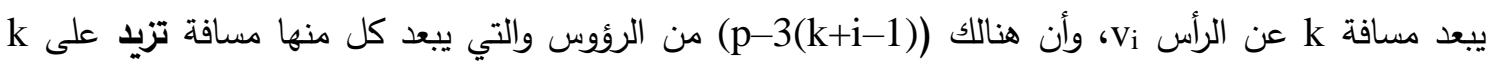

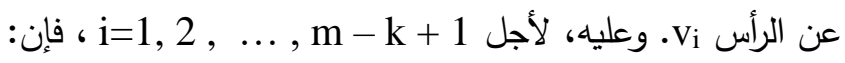

$\mathrm{C}_{\mathrm{n}}\left(\mathrm{v}_{\mathrm{i}}, \mathrm{PC}_{\mathrm{m}} ; \mathrm{k}\right)=\left(\begin{array}{c}\mathrm{p}-3(\mathrm{k}+\mathrm{i}-2) \\ \mathrm{n}-1\end{array}\right)-\left(\begin{array}{c}\mathrm{p}-3(\mathrm{k}+\mathrm{i}-1) \\ \mathrm{n}-1\end{array}\right)$

فضلاً عن ذلك فإن هنالك رأسان هما u2m, u2m+1 كل منهما يبعد مسافة k عن الرأس Vum-k+2 ولا يوجد أي

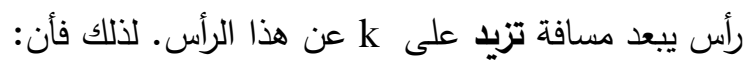

$\mathrm{C}_{\mathrm{n}}\left(\mathrm{v}_{\mathrm{m}-\mathrm{k}+2}, \mathrm{PC}_{\mathrm{m}}, \mathrm{k}\right)=\left(\begin{array}{c}2 \\ \mathrm{n}-1\end{array}\right)$

واضح أنه بسبب تماثل PC لكل

$\mathrm{C}_{\mathrm{n}}\left(\mathrm{V}, \mathrm{PC}_{\mathrm{m}}, \mathrm{k}\right)=2 \sum_{\mathrm{i}=1}^{\mathrm{m}-\mathrm{k}+1}\left[\left(\begin{array}{c}\mathrm{p}-3(\mathrm{k}+\mathrm{i}-2) \\ \mathrm{n}-1\end{array}\right)-\left(\begin{array}{c}\mathrm{p}-3(\mathrm{k}+\mathrm{i}-1) \\ \mathrm{n}-1\end{array}\right)\right]+2\left(\begin{array}{c}2 \\ \mathrm{n}-1\end{array}\right)$

,$=2\left[\left(\begin{array}{c}\mathrm{p}-3 \mathrm{k}+3 \\ \mathrm{n}-1\end{array}\right)-\left(\begin{array}{c}2 \\ \mathrm{n}-1\end{array}\right)\right]+2\left(\begin{array}{c}2 \\ \mathrm{n}-1\end{array}\right)=2\left(\begin{array}{c}\mathrm{p}-3 \mathrm{k}+3 \\ \mathrm{n}-1\end{array}\right)$

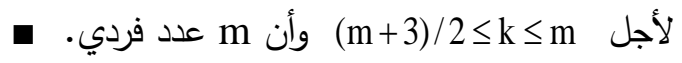

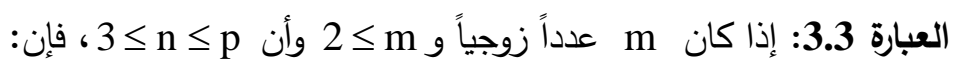

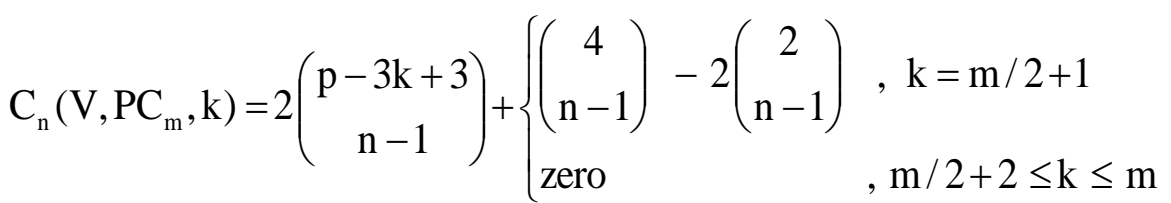

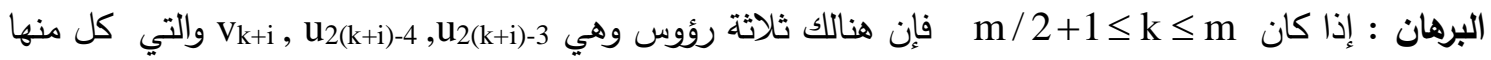

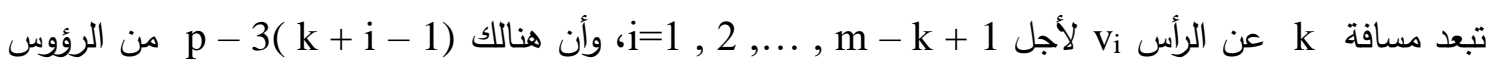

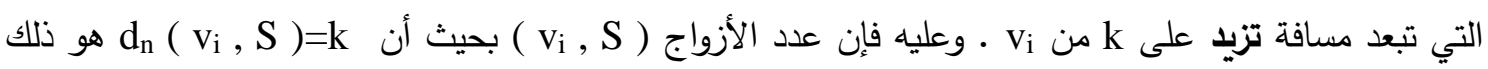

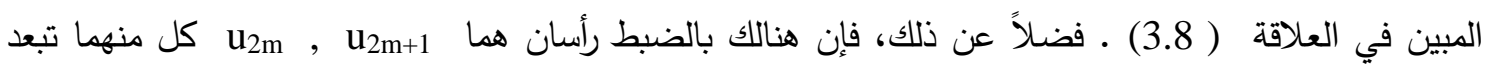

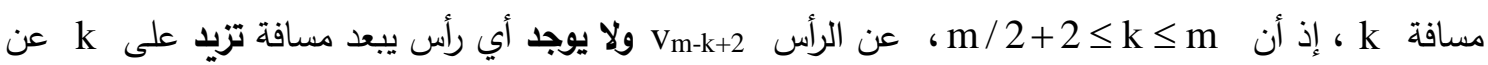

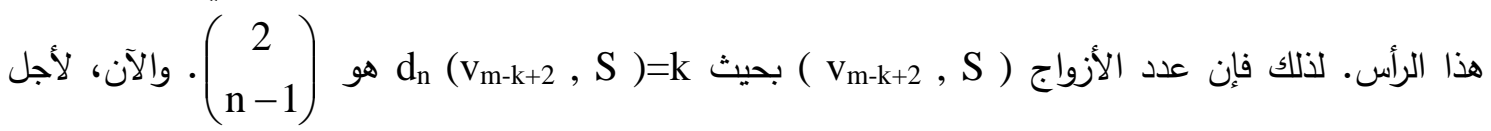
m توجد أربعة رؤوس وهي m/2+1 ولا يوجد أي رأس يبعد بمسافة تزيد على $V_{m / 2+1}$ $\mathrm{C}_{\mathrm{n}}\left(\mathrm{v}_{\mathrm{m} / 2+1}, \mathrm{PC}_{\mathrm{m}}, \mathrm{m} / 2+1\right)=\left(\begin{array}{c}4 \\ \mathrm{n}-1\end{array}\right)$

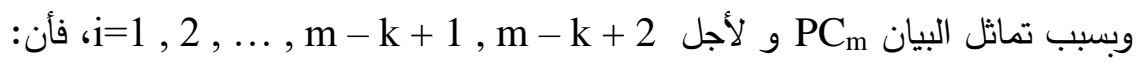
$\mathrm{C}_{\mathrm{n}}\left(\mathrm{v}_{\mathrm{i}}, \mathrm{PC}_{\mathrm{m}}, \mathrm{k}\right)=\mathrm{C}_{\mathrm{n}}\left(\mathrm{v}_{\mathrm{m}-\mathrm{i}+2}, \mathrm{PC}_{\mathrm{m}}, \mathrm{k}\right)$. 


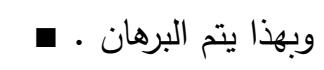

$\mathrm{C}_{\mathrm{n}}\left(\mathrm{V}, \mathrm{PC}_{\mathrm{m}}, \mathrm{m}+1\right)=2\left(\begin{array}{c}2 \\ \mathrm{n}-1\end{array}\right)$

يمكن أن نستنتج من ملاحظة الشكل 2.1 أن:

سواءً كان m فردياً أو زوجياً . لذلك من العبارات 3.1 و 3.2 و 3.3 نحصل على النتيجة الآتية:

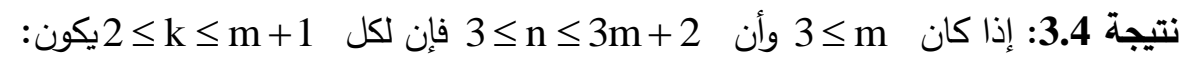
$\mathrm{C}_{\mathrm{n}}\left(\mathrm{V}, \mathrm{PC}_{\mathrm{m}}, \mathrm{k}\right)=2\left(\begin{array}{c}\mathrm{p}-3 \mathrm{k}+3 \\ \mathrm{n}-1\end{array}\right)+\mathrm{R}$

$\mathrm{R}=\left\{\begin{array}{r}(\mathrm{m}-2 \mathrm{k}+3)\left(\begin{array}{c}\mathrm{p}-6 \mathrm{k}+8 \\ \mathrm{n}-1\end{array}\right)-(\mathrm{m}-2 \mathrm{k}+1)\left(\begin{array}{c}\mathrm{p}-6 \mathrm{k}+2 \\ \mathrm{n}-1\end{array}\right)-2\left(\begin{array}{c}\mathrm{p}-6 \mathrm{k}+3 \\ \mathrm{n}-1\end{array}\right)-2\left(\begin{array}{c}\mathrm{p}-6 \mathrm{k}+6 \\ \mathrm{n}-1\end{array}\right), \\ 2 \leq \mathrm{k} \leq\lfloor(\mathrm{m}+1) / 2\rfloor \\ \left(\begin{array}{c}4 \\ \mathrm{n}-1\end{array}\right)-2\left(\begin{array}{c}2 \\ \mathrm{n}-1\end{array}\right), \\ \text { zero } \mathrm{k}=\frac{\mathrm{m}}{2}+1\end{array}\right.$ حيث أن:

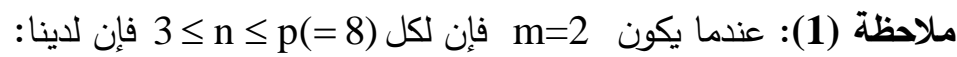
$\mathrm{C}_{\mathrm{n}}\left(\mathrm{V}, \mathrm{PC}_{2}, 2\right)=2\left(\begin{array}{c}5 \\ \mathrm{n}-1\end{array}\right)+\left(\begin{array}{c}4 \\ \mathrm{n}-1\end{array}\right), \mathrm{C}_{\mathrm{n}}\left(\mathrm{V}, \mathrm{PC}_{2}, 3\right)=2\left(\begin{array}{c}2 \\ \mathrm{n}-1\end{array}\right)$.

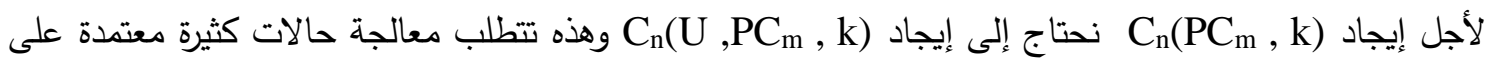

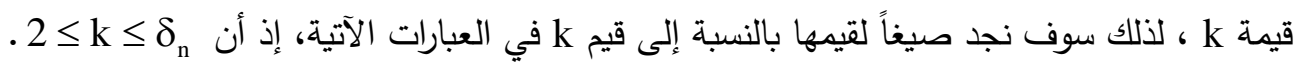

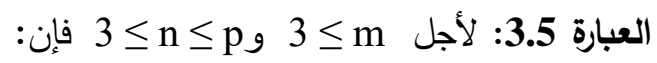
$\mathrm{C}_{\mathrm{n}}\left(\mathrm{U}, \mathrm{PC}_{\mathrm{m}}, 2\right)=(\mathrm{m}+2)\left(\begin{array}{l}\mathrm{p}-3 \\ \mathrm{n}-1\end{array}\right)+(\mathrm{m}-1)\left[\left(\begin{array}{l}\mathrm{p}-4 \\ \mathrm{n}-1\end{array}\right)-\left(\begin{array}{l}\mathrm{p}-8 \\ \mathrm{n}-1\end{array}\right)\right]-(\mathrm{m}-2)\left(\begin{array}{l}\mathrm{p}-7 \\ \mathrm{n}-1\end{array}\right)-2\left[\left(\begin{array}{l}\mathrm{p}-5 \\ \mathrm{n}-1\end{array}\right)+\left(\begin{array}{l}\mathrm{p}-6 \\ \mathrm{n}-1\end{array}\right)\right]$

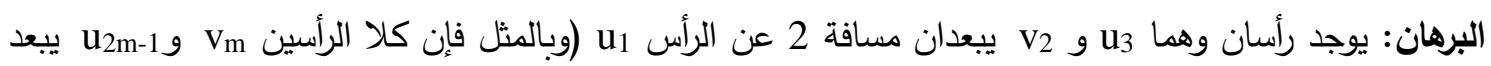

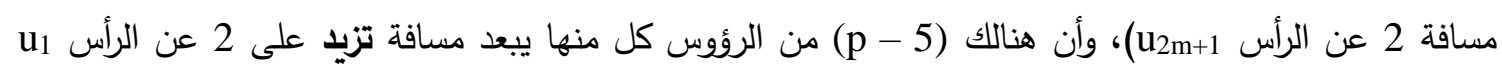
$\mathrm{C}_{\mathrm{n}}\left(\mathrm{u}_{\mathrm{i}}, \mathrm{PC}_{\mathrm{m}}, 2\right)=\left(\begin{array}{l}\mathrm{p}-3 \\ \mathrm{n}-1\end{array}\right)-\left(\begin{array}{l}\mathrm{p}-5 \\ \mathrm{n}-1\end{array}\right) \quad, \mathrm{i}=1,2 \mathrm{~m}+1$

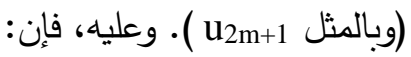

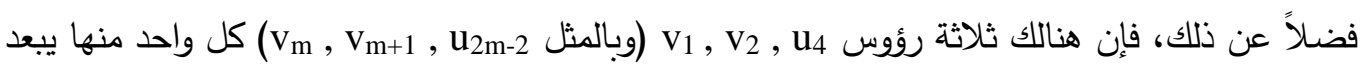

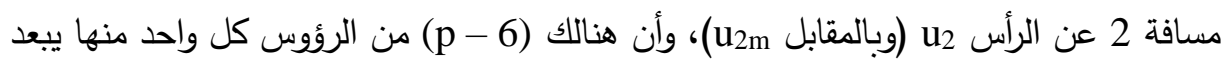

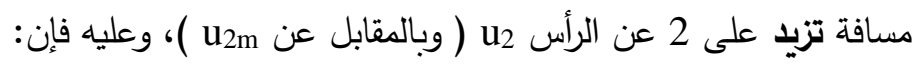
$C_{n}\left(u_{i}, P C_{m}, 2\right)=\left(\begin{array}{l}p-3 \\ n-1\end{array}\right)-\left(\begin{array}{l}p-6 \\ n-1\end{array}\right) \quad, i=2,2 m$. 
وأخيرا هنالك أربعة رؤوس وهي vل منها يبعد مسافة 2 عن الرأس

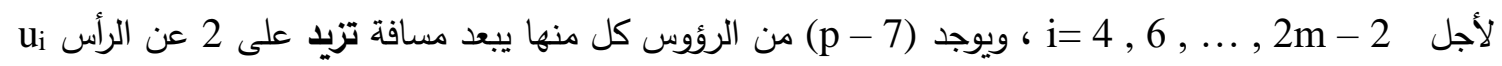
وعليه، فإن:

$\mathrm{C}_{\mathrm{n}}\left(\mathrm{u}_{\mathrm{i}}, \mathrm{PC}_{\mathrm{m}}, 2\right)=\left(\begin{array}{l}\mathrm{p}-3 \\ \mathrm{n}-1\end{array}\right)-\left(\begin{array}{l}\mathrm{p}-7 \\ \mathrm{n}-1\end{array}\right) \quad, \mathrm{i}=4,6, \ldots, 2 \mathrm{~m}-2$.

وكذلك توجد أربعة رؤوس وهي 1

$\mathrm{C}_{\mathrm{n}}\left(\mathrm{u}_{\mathrm{i}}, \mathrm{PC}_{\mathrm{m}}, 2\right)=\left(\begin{array}{l}\mathrm{p}-4 \\ \mathrm{n}-1\end{array}\right)-\left(\begin{array}{l}\mathrm{p}-8 \\ \mathrm{n}-1\end{array}\right), \mathrm{i}=3,5, \ldots, 2 \mathrm{~m}-1$.

وفي النهاية من (3.13) ، (3.14) ، (3.15) ، (3.16) وبجمع المقادير نحصل على المقدار (3.12) • وبهذا يتم البرهان. ملاحظة (2): إذا كان $3 \leq n \leq p$ فإنه عندما m=2 يكون لدينا: $\mathrm{C}_{\mathrm{n}}\left(\mathrm{U}, \mathrm{PC}_{2}, 2\right)=4\left(\begin{array}{c}5 \\ \mathrm{n}-1\end{array}\right)+\left(\begin{array}{c}4 \\ \mathrm{n}-1\end{array}\right)-2\left(\begin{array}{c}3 \\ \mathrm{n}-1\end{array}\right)-2\left(\begin{array}{c}2 \\ \mathrm{n}-1\end{array}\right)$. العبارة 3.6 : لأجل $3 \leq \mathrm{n} \leq \mathrm{p}$ ، 4 ، فإن: $\mathrm{C}_{\mathrm{n}}\left(\mathrm{U}, \mathrm{PC}_{\mathrm{m}}, 3\right)=(\mathrm{m}-4)\left(\begin{array}{l}\mathrm{p}-7 \\ \mathrm{n}-1\end{array}\right)+(\mathrm{m}-3)\left[\left(\begin{array}{l}\mathrm{p}-8 \\ \mathrm{n}-1\end{array}\right)-\left(\begin{array}{l}\mathrm{p}-12 \\ \mathrm{n}-1\end{array}\right)\right]$

$$
+2\left(\begin{array}{l}
p-5 \\
n-1
\end{array}\right)+2\left(\begin{array}{l}
p-6 \\
n-1
\end{array}\right)-(m-2)\left(\begin{array}{l}
p-11 \\
n-1
\end{array}\right)-2\left(\begin{array}{l}
p-10 \\
n-1
\end{array}\right)
$$

البرهان : يوجد رأسان وهما uli+3

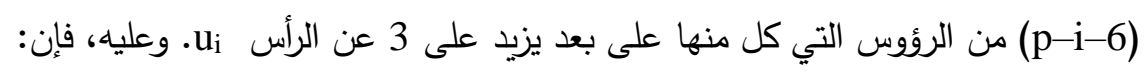
$\mathrm{C}_{\mathrm{n}}\left(\mathrm{u}_{\mathrm{i}}, \mathrm{PC}_{\mathrm{m}}, 3\right)=\left(\begin{array}{c}\mathrm{p}-\mathrm{i}-4 \\ \mathrm{n}-1\end{array}\right)-\left(\begin{array}{c}\mathrm{p}-\mathrm{i}-6 \\ \mathrm{n}-1\end{array}\right), \mathrm{i}=1,2$

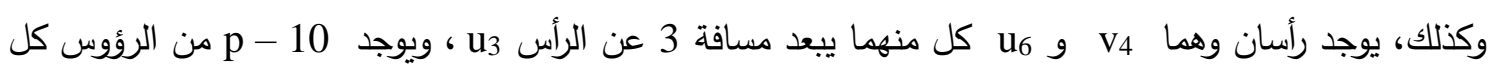

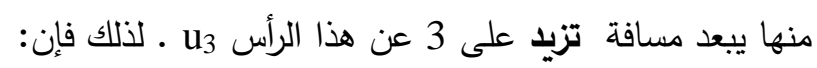
$\mathrm{C}_{\mathrm{n}}\left(\mathrm{u}_{3}, \mathrm{PC}_{\mathrm{m}}, 3\right)=\left(\begin{array}{c}\mathrm{p}-8 \\ \mathrm{n}-1\end{array}\right)-\left(\begin{array}{c}\mathrm{p}-10 \\ \mathrm{n}-1\end{array}\right)$

وبسبب التماثل في البيان PC ، فإنه لأجل i=1,2,3 نلاحظ أن: $\mathrm{C}_{\mathrm{n}}\left(\mathrm{u}_{\mathrm{i}}, \mathrm{PC}_{\mathrm{m}}, 3\right)=\mathrm{C}_{\mathrm{n}}\left(\mathrm{u}_{2 \mathrm{~m}-\mathrm{i}+2}, \mathrm{PC}_{\mathrm{m}}, 3\right)$ وهكذا، نحصل من (3.18) و (3.19) على: $\sum_{i \in \alpha} C_{n}\left(u_{i}, P C_{m}, 3\right)==2\left[\left(\begin{array}{l}p-5 \\ n-1\end{array}\right)+\left(\begin{array}{l}p-6 \\ n-1\end{array}\right)-\left(\begin{array}{l}p-7 \\ n-1\end{array}\right)-\left(\begin{array}{l}p-10 \\ n-1\end{array}\right)\right]$. $\alpha=\{1,2,3,2 \mathrm{~m}-1,2 \mathrm{~m}, 2 \mathrm{~m}+1\}$ إذ أن 


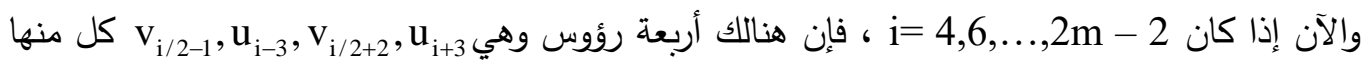

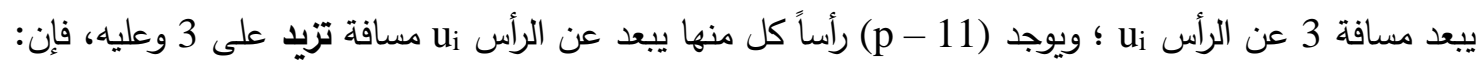
$\mathrm{C}_{\mathrm{n}}\left(\mathrm{u}_{\mathrm{i}}, \mathrm{PC}_{\mathrm{m}}, 3\right)=\left(\begin{array}{c}\mathrm{p}-7 \\ \mathrm{n}-1\end{array}\right)-\left(\begin{array}{c}\mathrm{p}-11 \\ \mathrm{n}-1\end{array}\right), \mathrm{i}=4,6, \ldots, 2 \mathrm{~m}-2$.

كذلك، توجد أربعة رؤوس هي i

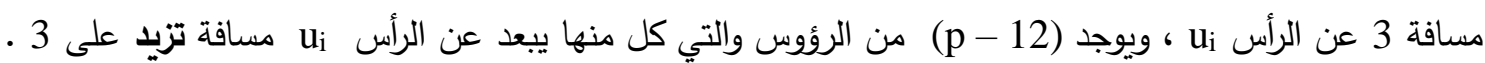
$C_{n}\left(u_{i}, P C_{m}, 3\right)=\left(\begin{array}{c}p-8 \\ n-1\end{array}\right)-\left(\begin{array}{c}p-12 \\ n-1\end{array}\right), i=5,7, \ldots, 2 m-3$ وعليه، فإن: قوأخيراً، من (3.20) و(3.21) و(3.22) وبالجمع والتبسيط نحصل على (3.17) وبهذا يتم البرهان. ولإكمال حالات كون k=3 بالنسبة لقيم m=3 وm=3 نعطي الملاحظة الآتية: ملاحظة (3): إذا كان 3 s 3 فإن: $\mathrm{C}_{\mathrm{n}}\left(\mathrm{U}, \mathrm{PC}_{2}, 3\right)=2\left(\begin{array}{c}3 \\ \mathrm{n}-1\end{array}\right)+2\left(\begin{array}{c}2 \\ \mathrm{n}-1\end{array}\right)$
$\mathrm{C}_{\mathrm{n}}\left(\mathrm{U}, \mathrm{PC}_{3}, 3\right)=2\left(\begin{array}{c}6 \\ \mathrm{n}-1\end{array}\right)+2\left(\begin{array}{c}5 \\ \mathrm{n}-1\end{array}\right)-\left(\begin{array}{c}4 \\ \mathrm{n}-1\end{array}\right)$.

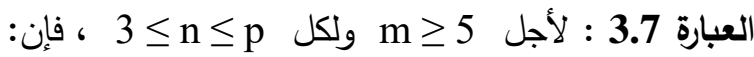

$$
\begin{aligned}
\mathrm{C}_{\mathrm{n}}\left(\mathrm{U}, \mathrm{PC}_{\mathrm{m}}, 4\right)=(\mathrm{m}-2)\left[\left(\begin{array}{c}
\mathrm{p}-11 \\
\mathrm{n}-1
\end{array}\right)\right. & \left.-\left(\begin{array}{c}
\mathrm{p}-15 \\
\mathrm{n}-1
\end{array}\right)\right]+(\mathrm{m}-5)\left[\left(\begin{array}{c}
\mathrm{p}-12 \\
\mathrm{n}-1
\end{array}\right)-\left(\begin{array}{c}
\mathrm{p}-16 \\
\mathrm{n}-1
\end{array}\right)\right] \\
& +2\left[\left(\begin{array}{c}
\mathrm{p}-7 \\
\mathrm{n}-1
\end{array}\right)+\left(\begin{array}{c}
\mathrm{p}-8 \\
\mathrm{n}-1
\end{array}\right)\right]-2\left[\left(\begin{array}{c}
\mathrm{p}-9 \\
\mathrm{n}-1
\end{array}\right)+\left(\begin{array}{c}
\mathrm{p}-13 \\
\mathrm{n}-1
\end{array}\right)\right]
\end{aligned}
$$

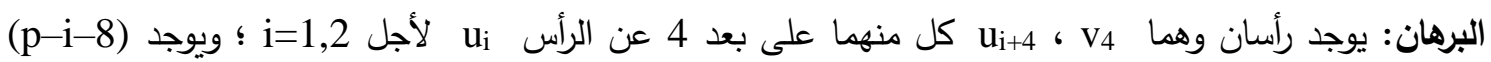

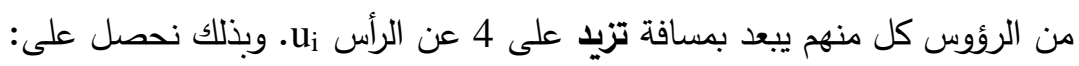
$C_{n}\left(u_{i}, P C_{m}, 4\right)=\left(\begin{array}{c}p-i-6 \\ n-1\end{array}\right)-\left(\begin{array}{c}p-i-8 \\ n-1\end{array}\right), i=1,2$.

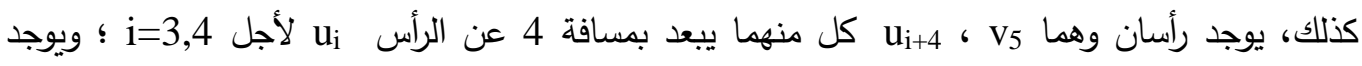
(p-i-9) $\mathrm{C}_{\mathrm{n}}\left(\mathrm{u}_{\mathrm{i}}, \mathrm{PC}_{\mathrm{m}}, 4\right)=\left(\begin{array}{c}\mathrm{p}-\mathrm{i}-7 \\ \mathrm{n}-1\end{array}\right)-\left(\begin{array}{c}\mathrm{p}-\mathrm{i}-9 \\ \mathrm{n}-1\end{array}\right), \mathrm{i}=3,4$.

إضافة إلى ذلك، يوجد ثلاثة رؤوس وهي un , u9, v6 كل منها يبعد مسافة 4 عن الرأس un ؛ ويوجد (15-p)

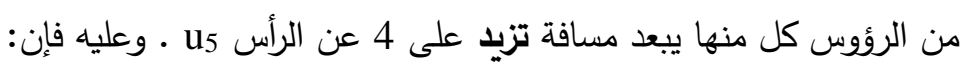


$\mathrm{C}_{\mathrm{n}}\left(\mathrm{u}_{5}, \mathrm{PC}_{\mathrm{m}}, 4\right)=\left(\begin{array}{c}\mathrm{p}-12 \\ \mathrm{n}-1\end{array}\right)-\left(\begin{array}{c}\mathrm{p}-15 \\ \mathrm{n}-1\end{array}\right)$

وبسبب تماثل البيان PC Pإن لدينا:

$\mathrm{C}_{\mathrm{n}}\left(\mathrm{u}_{\mathrm{i}}, \mathrm{PC}_{\mathrm{m}}, 4\right)=\mathrm{C}_{\mathrm{n}}\left(\mathrm{u}_{2 \mathrm{~m}-\mathrm{i}+2}, \mathrm{PC}_{\mathrm{m}}, 4\right), \quad \mathrm{i}=1,2,3,4,5$.

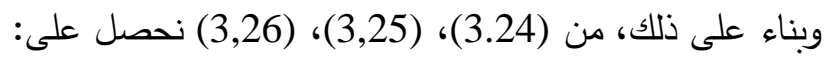

$\sum_{i \in \beta} C_{n}\left(u_{i}, P C_{m}, 4\right)=2\left[\left(\begin{array}{c}p-7 \\ n-1\end{array}\right)+\left(\begin{array}{c}p-8 \\ n-1\end{array}\right)+\left(\begin{array}{c}p-11 \\ n-1\end{array}\right)\right]-2\left[\left(\begin{array}{c}p-9 \\ n-1\end{array}\right)+\left(\begin{array}{c}p-13 \\ n-1\end{array}\right)+\left(\begin{array}{c}p-15 \\ n-1\end{array}\right)\right]$

إذ أن:

$\beta=\{1,2,3,4,5,2 \mathrm{~m}-3,2 \mathrm{~m}-2,2 \mathrm{~m}-1,2 \mathrm{~m}, 2 \mathrm{~m}+1\}$.

والآن، إذا كان i=6, il= فإن هنالك بالضبط أربعة رؤوس وهي

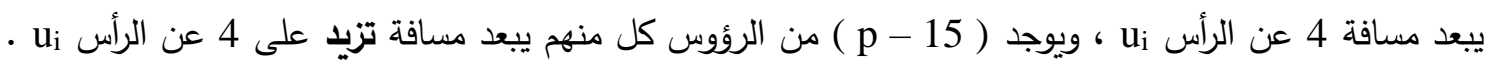
لذلك، فإن:

$\mathrm{C}_{\mathrm{n}}\left(\mathrm{u}_{\mathrm{i}}, \mathrm{PC}_{\mathrm{m}}, 4\right)=\left(\begin{array}{c}\mathrm{p}-11 \\ \mathrm{n}-1\end{array}\right)-\left(\begin{array}{c}\mathrm{p}-15 \\ \mathrm{n}-1\end{array}\right), \quad \mathrm{i}=6,8, \ldots, 2 \mathrm{~m}-4$

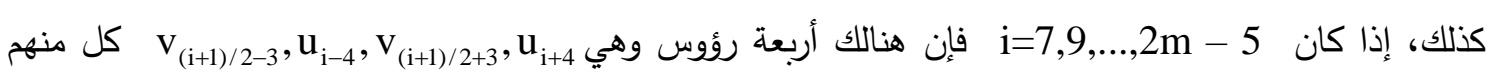
يبعد مسافة 4 عن الرأس uni ويوجد بالضبط ( 16 - p ) من الرؤوس كل منهم يبعد مسافة تزيد على 4 عن الرأس u . لذلك، فإن:

$\mathrm{C}_{\mathrm{n}}\left(\mathrm{u}_{\mathrm{i}}, \mathrm{PC}_{\mathrm{m}}, 4\right)=\left(\begin{array}{c}\mathrm{p}-12 \\ \mathrm{n}-1\end{array}\right)-\left(\begin{array}{c}\mathrm{p}-16 \\ \mathrm{n}-1\end{array}\right), \mathrm{i}=7,9, \ldots, 2 \mathrm{~m}-5$

صأخيراً من (3.27) و(3.28) و(3.29) وبإجراء بعض الاختصار نحصل على (3.23)، وبهذا يتم البرهان. ل ملاحظة (4): إذا كان ns n 3 فإن لقيم m=2,3,4 نحصل مباشرة على:

$\mathrm{C}_{\mathrm{n}}\left(\mathrm{U}, \mathrm{PC}_{2}, 4\right)=0$,

$\mathrm{C}_{\mathrm{n}}\left(\mathrm{U}, \mathrm{PC}_{3}, 4\right)=2\left[\left(\begin{array}{c}4 \\ \mathrm{n}-1\end{array}\right)+\left(\begin{array}{c}3 \\ \mathrm{n}-1\end{array}\right)-\left(\begin{array}{c}2 \\ \mathrm{n}-1\end{array}\right)\right]$,

$\mathrm{C}_{\mathrm{n}}\left(\mathrm{U}, \mathrm{PC}_{4}, 4\right)=2\left[\left(\begin{array}{c}7 \\ \mathrm{n}-1\end{array}\right)+\left(\begin{array}{c}6 \\ \mathrm{n}-1\end{array}\right)+\left(\begin{array}{c}3 \\ \mathrm{n}-1\end{array}\right)-\left(\begin{array}{c}5 \\ \mathrm{n}-1\end{array}\right)\right]-\left(\begin{array}{c}2 \\ \mathrm{n}-1\end{array}\right)$.

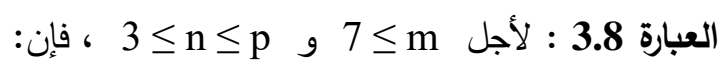

$\mathrm{C}_{\mathrm{n}}\left(\mathrm{U}, \mathrm{PC}_{\mathrm{m}}, 5\right)=(\mathrm{m}-5)\left(\begin{array}{l}\mathrm{p}-16 \\ \mathrm{n}-1\end{array}\right)+(\mathrm{m}-6)\left[\left(\begin{array}{l}\mathrm{p}-15 \\ \mathrm{n}-1\end{array}\right)-\left(\begin{array}{l}\mathrm{p}-19 \\ \mathrm{n}-1\end{array}\right)\right]+2\left[\left(\begin{array}{l}\mathrm{p}-9 \\ \mathrm{n}-1\end{array}\right)+\left(\begin{array}{l}\mathrm{p}-10 \\ \mathrm{n}-1\end{array}\right)+\left(\begin{array}{l}\mathrm{p}-13 \\ \mathrm{n}-1\end{array}\right)\right]$ $-(\mathrm{m}-7)\left(\begin{array}{l}\mathrm{p}-22 \\ \mathrm{n}-1\end{array}\right)-2\left[\left(\begin{array}{l}\mathrm{p}-12 \\ \mathrm{n}-1\end{array}\right)+2\left(\begin{array}{l}\mathrm{p}-18 \\ \mathrm{n}-1\end{array}\right)+\left(\begin{array}{l}\mathrm{p}-21 \\ \mathrm{n}-1\end{array}\right)\right]$.

البرهان: توجد ثلاثة رؤوس وهي

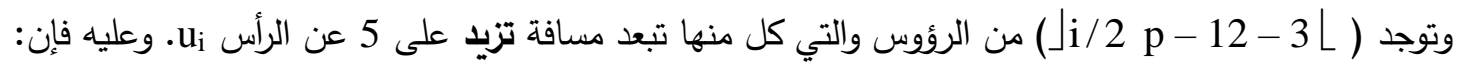


$\mathrm{C}_{\mathrm{n}}\left(\mathrm{u}_{\mathrm{i}}, \mathrm{PC}_{\mathrm{m}}, 5\right)=\left(\begin{array}{c}\mathrm{p}-9-3\lfloor\mathrm{i} / 2\rfloor \\ \mathrm{n}-1\end{array}\right)-\left(\begin{array}{c}\mathrm{p}-12-3\lfloor\mathrm{i} / 2\rfloor \\ \mathrm{n}-1\end{array}\right), \mathrm{i}=1,3,5$.

فضـلاً عن ذلك يوجد رأسـان وهمـا

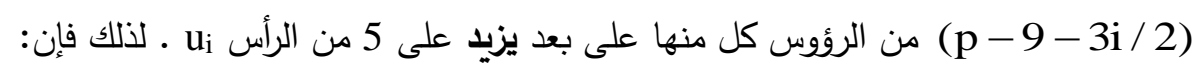

$\mathrm{C}_{\mathrm{n}}\left(\mathrm{u}_{\mathrm{i}}, \mathrm{PC}_{\mathrm{m}}, 5\right)=\left(\begin{array}{c}\mathrm{p}-7-3 \mathrm{i} / 2 \\ \mathrm{n}-1\end{array}\right)-\left(\begin{array}{c}\mathrm{p}-9-3 \mathrm{i} / 2 \\ \mathrm{n}-1\end{array}\right), \mathrm{i}=2,4$.

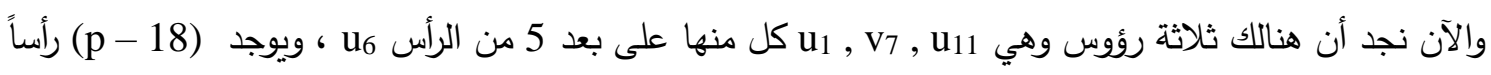

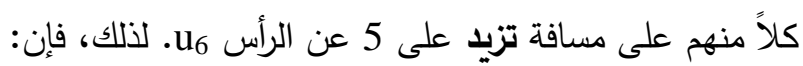

$\mathrm{C}_{\mathrm{n}}\left(\mathrm{u}_{6}, \mathrm{PC}_{\mathrm{m}}, 5\right)=\left(\begin{array}{c}\mathrm{p}-15 \\ \mathrm{n}-1\end{array}\right)-\left(\begin{array}{c}\mathrm{p}-18 \\ \mathrm{n}-1\end{array}\right)$.

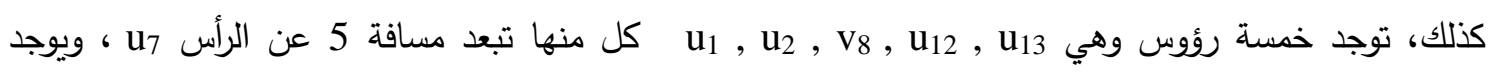

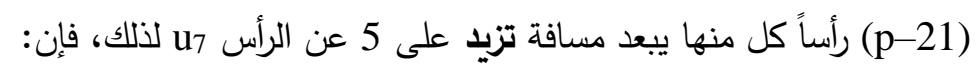

$\mathrm{C}_{\mathrm{n}}\left(\mathrm{u}_{7}, \mathrm{PC}_{\mathrm{m}}, 5\right)=\left(\begin{array}{c}\mathrm{p}-16 \\ \mathrm{n}-1\end{array}\right)-\left(\begin{array}{c}\mathrm{p}-21 \\ \mathrm{n}-1\end{array}\right)$

$\mathrm{C}_{\mathrm{n}}\left(\mathrm{u}_{\mathrm{i}}, \mathrm{PC}_{\mathrm{m}}, 5\right)=\mathrm{C}_{\mathrm{n}}\left(\mathrm{u}_{2 \mathrm{~m}-\mathrm{i}-2}, \mathrm{PC}_{\mathrm{m}}, 5\right), \quad \mathrm{i}=1,2,3, \ldots, 7$

وبسبب احتواء PC على تماثل معين فإن:

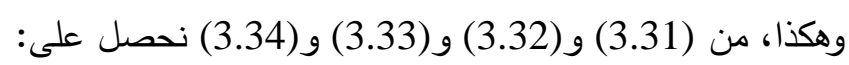

$\sum_{i \in \gamma} C_{n}\left(u_{i}, P C_{m}, 5\right)=2\left[\left(\begin{array}{c}p-9 \\ n-1\end{array}\right)+\left(\begin{array}{c}p-10 \\ n-1\end{array}\right)+\left(\begin{array}{c}p-13 \\ n-1\end{array}\right)+\left(\begin{array}{c}p-16 \\ n-1\end{array}\right)-\left(\begin{array}{c}p-12 \\ n-1\end{array}\right)-2\left(\begin{array}{c}p-18 \\ n-1\end{array}\right)-\left(\begin{array}{c}p-21 \\ n-1\end{array}\right)\right]$

حيث أن:

$\gamma=\{1,2,3,4,5,6,7,2 \mathrm{~m}-5,2 \mathrm{~m}-4,2 \mathrm{~m}-3,2 \mathrm{~m}-2,2 \mathrm{~m}-1,2 \mathrm{~m}, 2 \mathrm{~m}+1\}$

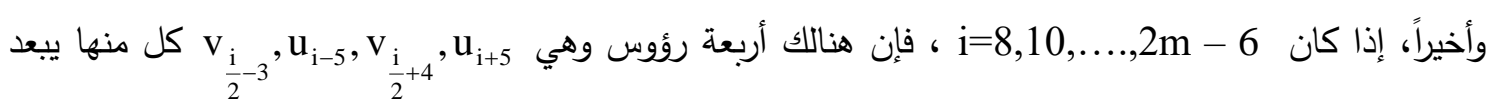
مسافة 5 عن الرأس ui . ويوجد (19 - p ) رأساً كل منها يبعد مسافة تزيد على 5 عن الرأس لذلك فإن: $\mathrm{C}_{\mathrm{n}}\left(\mathrm{u}_{\mathrm{i}}, \mathrm{PC}_{\mathrm{m}}, 5\right)=\left(\begin{array}{c}\mathrm{p}-15 \\ \mathrm{n}-1\end{array}\right)-\left(\begin{array}{c}\mathrm{p}-19 \\ \mathrm{n}-1\end{array}\right) . \mathrm{i}=8,10, \ldots ., 2 \mathrm{~m}-6$.

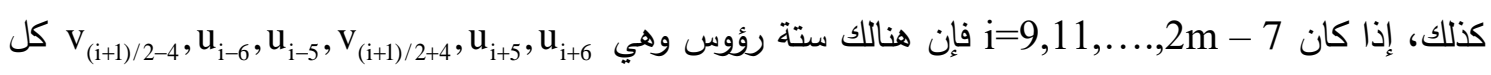

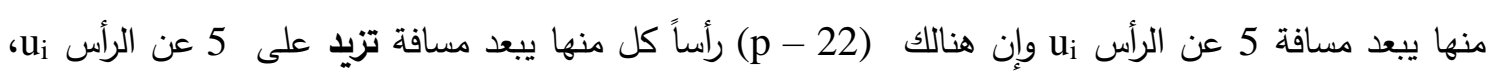
لذلك فإن: $\mathrm{C}_{\mathrm{n}}\left(\mathrm{u}_{\mathrm{i}}, \mathrm{PC}_{\mathrm{m}}, 5\right)=\left(\begin{array}{c}\mathrm{p}-16 \\ \mathrm{n}-1\end{array}\right)-\left(\begin{array}{c}\mathrm{p}-22 \\ \mathrm{n}-1\end{array}\right) . \mathrm{i}=9,11, \ldots, 2 \mathrm{~m}-7$.

قأخيراً، من (3.35) و(3.36) و(3.37) وبالجمع والتبسيط نحصل على (3.30)، وبهذا يتم البرهان. ملاحظة (5): إذا كان 3 s 3 فإن: 


$$
\begin{aligned}
& \mathrm{C}_{\mathrm{n}}\left(\mathrm{U}, \mathrm{PC}_{2}, 5\right)=0, \mathrm{C}_{\mathrm{n}}\left(\mathrm{U}, \mathrm{PC}_{3}, 5\right)=2\left(\begin{array}{c}
2 \\
\mathrm{n}-1
\end{array}\right), \\
& \mathrm{C}_{\mathrm{n}}\left(\mathrm{U}, \mathrm{PC}_{4}, 5\right)=2\left[\left(\begin{array}{c}
5 \\
\mathrm{n}-1
\end{array}\right)+\left(\begin{array}{c}
4 \\
\mathrm{n}-1
\end{array}\right)-\left(\begin{array}{c}
2 \\
\mathrm{n}-1
\end{array}\right)\right], \\
& \mathrm{C}_{\mathrm{n}}\left(\mathrm{U}, \mathrm{PC}_{5}, 5\right)=2\left[\left(\begin{array}{c}
8 \\
\mathrm{n}-1
\end{array}\right)+\left(\begin{array}{c}
7 \\
\mathrm{n}-1
\end{array}\right)+\left(\begin{array}{c}
4 \\
\mathrm{n}-1
\end{array}\right)-\left(\begin{array}{c}
5 \\
\mathrm{n}-1
\end{array}\right)\right]-\left(\begin{array}{c}
2 \\
\mathrm{n}-1
\end{array}\right), \\
& \mathrm{C}_{\mathrm{n}}\left(\mathrm{U}, \mathrm{PC}_{6}, 5\right)=2\left[\left(\begin{array}{c}
11 \\
\mathrm{n}-1
\end{array}\right)+\left(\begin{array}{c}
10 \\
\mathrm{n}-1
\end{array}\right)+\left(\begin{array}{c}
7 \\
\mathrm{n}-1
\end{array}\right)-\left(\begin{array}{c}
8 \\
\mathrm{n}-1
\end{array}\right)\right]+\left(\begin{array}{c}
4 \\
\mathrm{n}-1
\end{array}\right)-4\left(\begin{array}{c}
2 \\
\mathrm{n}-1
\end{array}\right) .
\end{aligned}
$$

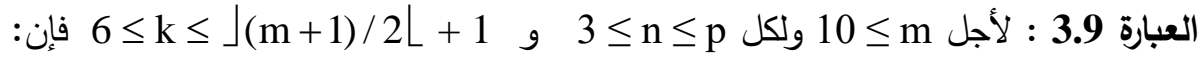$$
\mathrm{C}_{\mathrm{n}}\left(\mathrm{U}, \mathrm{PC}_{\mathrm{m}}, \mathrm{k}\right)=4\left(\begin{array}{c}
\mathrm{p}-3 \mathrm{k}+6 \\
\mathrm{n}-1
\end{array}\right)-4\left(\begin{array}{c}
\mathrm{p}-6 \mathrm{k}+12 \\
\mathrm{n}-1
\end{array}\right)-2\left(\begin{array}{c}
\mathrm{p}-6 \mathrm{k}+9 \\
\mathrm{n}-1
\end{array}\right)-2\left(\begin{array}{c}
\mathrm{p}-6 \mathrm{k}+15 \\
\mathrm{n}-1
\end{array}\right)
$$$$
\begin{aligned}
& -(\mathrm{m}-2 \mathrm{k}+3)\left(\begin{array}{c}
\mathrm{p}-6 \mathrm{k}+8 \\
\mathrm{n}-1
\end{array}\right)-(\mathrm{m}-2 \mathrm{k}+4)\left(\begin{array}{c}
\mathrm{p}-6 \mathrm{k}+11 \\
\mathrm{n}-1
\end{array}\right) \\
& +(\mathrm{m}-2 \mathrm{k}+5)\left(\begin{array}{c}
\mathrm{p}-6 \mathrm{k}+14 \\
\mathrm{n}-1
\end{array}\right)+(\mathrm{m}-2 \mathrm{k}+6)\left(\begin{array}{c}
\mathrm{p}-6 \mathrm{k}+17 \\
\mathrm{n}-1
\end{array}\right) .
\end{aligned}
$$

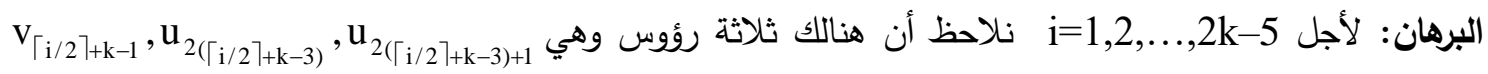

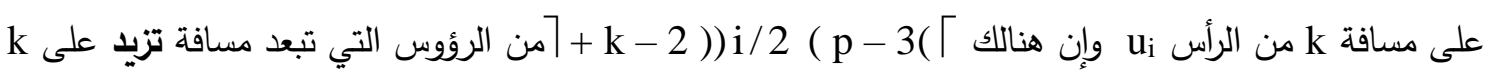

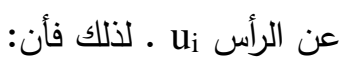

$$
\mathrm{C}_{\mathrm{n}}\left(\mathrm{u}_{\mathrm{i}}, \mathrm{PC}_{\mathrm{m}}, \mathrm{k}\right)=\left(\begin{array}{c}
\mathrm{p}+9-3 \mathrm{k}-3\lceil\mathrm{i} / 2\rceil \\
\mathrm{n}-1
\end{array}\right)-\left(\begin{array}{c}
\mathrm{p}+6-3 \mathrm{k}-3\lceil\mathrm{i} / 2\rceil \\
\mathrm{n}-1
\end{array}\right),
$$

$$
\text { ل كل ف }
$$

فضلاً عن ذلك، توجد خمسة رؤوس

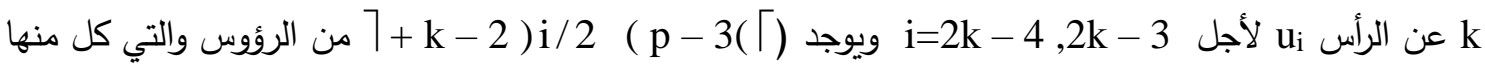

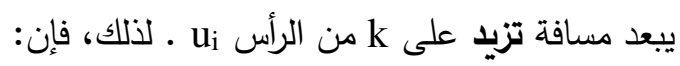

$\mathrm{C}_{\mathrm{n}}\left(\mathrm{u}_{\mathrm{i}}, \mathrm{PC}_{\mathrm{m}}, \mathrm{k}\right)=\left(\begin{array}{c}\mathrm{p}+11-3 \mathrm{k}-3\lceil\mathrm{i} / 2\rceil \\ \mathrm{n}-1\end{array}\right)-\left(\begin{array}{c}\mathrm{p}+6-3 \mathrm{k}-3\lceil\mathrm{i} / 2\rceil \\ \mathrm{n}-1\end{array}\right)$, لأجل i= 2k-4, 2k-3 - و وبما أن: $\mathrm{C}_{\mathrm{n}}\left(\mathrm{u}_{\mathrm{i}}, \mathrm{PC}_{\mathrm{m}}, \mathrm{k}\right)=\mathrm{C}_{\mathrm{n}}\left(\mathrm{u}_{2 \mathrm{~m}+2-\mathrm{i}}, \mathrm{PC}_{\mathrm{m}}, \mathrm{k}\right), \quad \mathrm{i}=1,2, \ldots ., 2 \mathrm{k}-3$

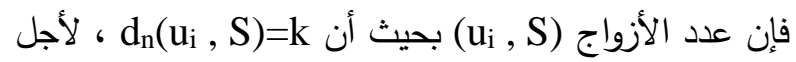
$\mathrm{i} \in\{1,2, \ldots, 2 \mathrm{k}-3 ; 2(\mathrm{~m}-\mathrm{k})+5,2(\mathrm{~m}-\mathrm{k})+6, \ldots ., 2 \mathrm{~m}+1\}, 6 \leq \mathrm{k} \leq\lfloor(\mathrm{m}+1) / 2\rfloor+1$ 


$$
\begin{aligned}
& 2 \sum_{\mathrm{i}=1}^{2 \mathrm{k}-5}\left[\left(\begin{array}{c}
\mathrm{p}+9-3 \mathrm{k}-3\lceil\mathrm{i} / 2\rceil \\
\mathrm{n}-1
\end{array}\right)-\left(\begin{array}{c}
\mathrm{p}+6-3 \mathrm{k}-3\lceil\mathrm{i} / 2\rceil \\
\mathrm{n}-1
\end{array}\right)\right] \\
& +2\left[\left(\begin{array}{c}
\mathrm{p}-6 \mathrm{k}+17 \\
\mathrm{n}-1
\end{array}\right)+\left(\begin{array}{c}
\mathrm{p}-6 \mathrm{k}+14 \\
\mathrm{n}-1
\end{array}\right)-\left(\begin{array}{c}
\mathrm{p}-6 \mathrm{k}+12 \\
\mathrm{n}-1
\end{array}\right)-\left(\begin{array}{c}
\mathrm{p}-6 \mathrm{k}+9 \\
\mathrm{n}-1
\end{array}\right)\right] \\
& =4\left(\begin{array}{c}
\mathrm{p}-3 \mathrm{k}+6 \\
\mathrm{n}-1
\end{array}\right)-2\left(\begin{array}{c}
\mathrm{p}-6 \mathrm{k}+15 \\
\mathrm{n}-1
\end{array}\right)-2\left(\begin{array}{c}
\mathrm{p}-6 \mathrm{k}+12 \\
\mathrm{n}-1
\end{array}\right) \\
& +2\left[\left(\begin{array}{c}
\mathrm{p}-6 \mathrm{k}+17 \\
\mathrm{n}-1
\end{array}\right)+\left(\begin{array}{c}
\mathrm{p}-6 \mathrm{k}+14 \\
\mathrm{n}-1
\end{array}\right)-\left(\begin{array}{c}
\mathrm{p}-6 \mathrm{k}+12 \\
\mathrm{n}-1
\end{array}\right)-\left(\begin{array}{c}
\mathrm{p}-6 \mathrm{k}+9 \\
\mathrm{n}-1
\end{array}\right)\right] \\
& =4\left(\begin{array}{c}
\mathrm{p}-3 \mathrm{k}+6 \\
\mathrm{n}-1
\end{array}\right)+2\left(\begin{array}{c}
\mathrm{p}-6 \mathrm{k}+14 \\
\mathrm{n}-1
\end{array}\right)+2\left(\begin{array}{c}
\mathrm{p}-6 \mathrm{k}+17 \\
\mathrm{n}-1
\end{array}\right)-2\left(\begin{array}{c}
\mathrm{p}-6 \mathrm{k}+9 \\
\mathrm{n}-1
\end{array}\right) \\
& -4\left(\begin{array}{c}
\mathrm{p}-6 \mathrm{k}+12 \\
\mathrm{n}-1
\end{array}\right)-2\left(\begin{array}{c}
\mathrm{p}-6 \mathrm{k}+15 \\
\mathrm{n}-1
\end{array}\right) .
\end{aligned}
$$

وأخيراً، فإنَ لكل i= 2k-2 , 2k,2k + 2,..,2m-2k+4 توجد ستة رؤوس وهي

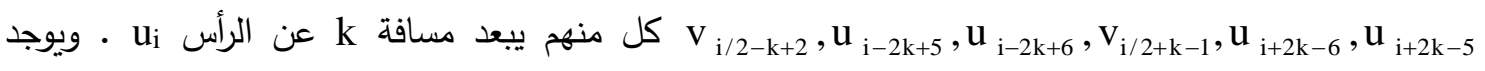
(p-6k+11) رأساً كل منهم يبعد مسافة تزيد على k عن الرأس un . لذلك، فإن: $\mathrm{C}_{\mathrm{n}}\left(\mathrm{u}_{\mathrm{i}}, \mathrm{PC}_{\mathrm{m}}, \mathrm{k}\right)=\left(\begin{array}{c}\mathrm{p}-6 \mathrm{k}+17 \\ \mathrm{n}-1\end{array}\right)-\left(\begin{array}{c}\mathrm{p}-6 \mathrm{k}+11 \\ \mathrm{n}-1\end{array}\right)$,

. $\mathrm{i}=2 \mathrm{k}-2,2 \mathrm{k}, 2 \mathrm{k}+2, \ldots, 2 \mathrm{~m}-2 \mathrm{k}+4$ كل كذلك، توجد ستة رؤوس وهي $\mathrm{v}_{(\mathrm{i}+1) / 2-k+1}, \mathrm{u}_{\mathrm{i}-2 \mathrm{k}+4}, \mathrm{u}_{\mathrm{i}-2 \mathrm{k}+5}, \mathrm{v}_{(\mathrm{i}+1) / 2+\mathrm{k}-1}, \mathrm{u}_{\mathrm{i}+2 \mathrm{k}-4}, \mathrm{u}_{\mathrm{i}+2 \mathrm{k}-5}$ مسافة k عن الرأس u لأجل un $\mathrm{C}_{\mathrm{n}}\left(\mathrm{u}_{\mathrm{i}}, \mathrm{PC}_{\mathrm{m}}, \mathrm{k}\right)=\left(\begin{array}{c}\mathrm{p}-6 \mathrm{k}+14 \\ \mathrm{n}-1\end{array}\right)-\left(\begin{array}{c}\mathrm{p}-6 \mathrm{k}+8 \\ \mathrm{n}-1\end{array}\right)$,

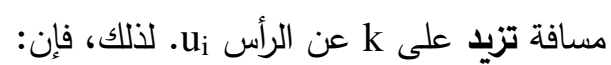

$$
\text { i=2k-1,2k + 1,..,2m-2k+3 لأجل }
$$

وفي الختام من (3.41) و (3.42) و (3.43) وبإجراء التبسيط المطلوب نحصل على (3.38). وبهذا

$$
\text { ص يتم البرهان. }
$$

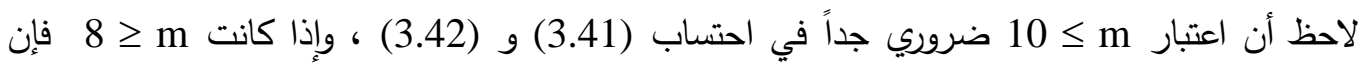

$$
\text { 5 2 k }
$$

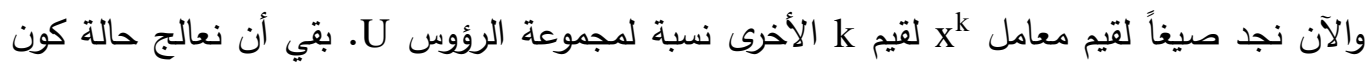

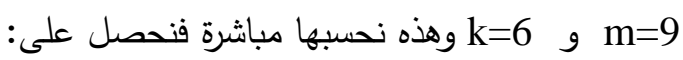
ملاحظة (6): إذا كان m=9 وكان k=6 فإن: 
$\mathrm{C}_{\mathrm{n}}\left(\mathrm{U}, \mathrm{PC}_{9}, 6\right)=4\left(\begin{array}{c}17 \\ \mathrm{n}-1\end{array}\right)+3\left(\begin{array}{c}10 \\ \mathrm{n}-1\end{array}\right)+2\left(\begin{array}{c}7 \\ \mathrm{n}-1\end{array}\right)-4\left(\begin{array}{c}5 \\ \mathrm{n}-1\end{array}\right)-\left(\begin{array}{c}4 \\ \mathrm{n}-1\end{array}\right)-2\left(\begin{array}{c}2 \\ \mathrm{n}-1\end{array}\right)-2\left(\begin{array}{c}8 \\ \mathrm{n}-1\end{array}\right)$.

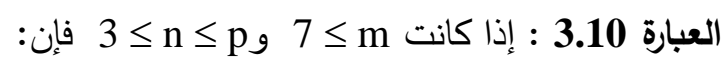

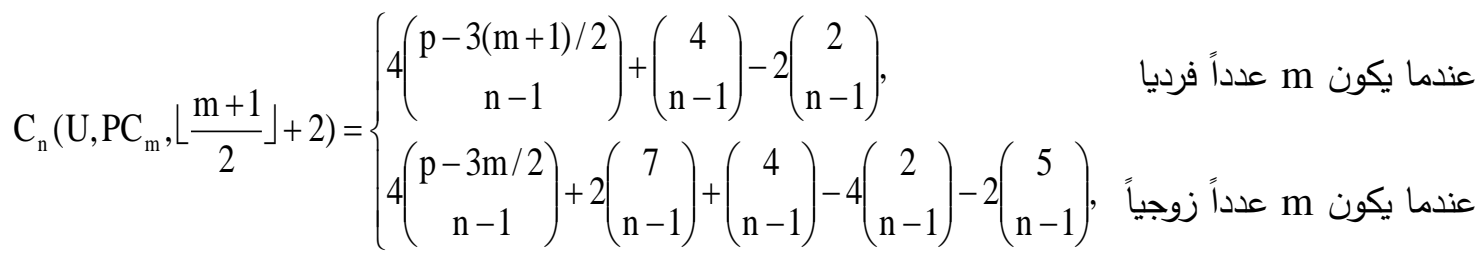

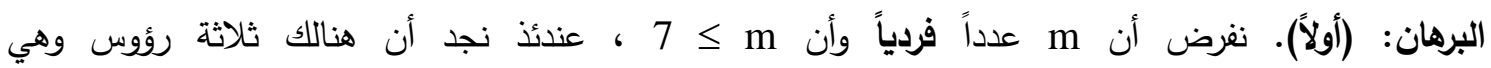
$u_{i}$ الرأس لأجل ili

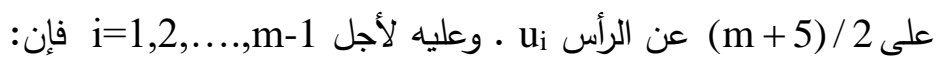
$\mathrm{C}_{\mathrm{n}}\left(\mathrm{u}_{\mathrm{i}}, \mathrm{PC}_{\mathrm{m}}, \frac{\mathrm{m}+5}{2}\right)=\left(\begin{array}{c}\mathrm{p}-3(\mathrm{~m}-1) / 2-3\lceil\mathrm{i} / 2\rceil \\ \mathrm{n}-1\end{array}\right)-\left(\begin{array}{c}\mathrm{p}-3(\mathrm{~m}+1) / 2-3\lceil\mathrm{i} / 2\rceil \\ \mathrm{n}-1\end{array}\right)$, وبما أن:

$\mathrm{C}_{\mathrm{n}}\left(\mathrm{u}_{\mathrm{i}}, \mathrm{PC}_{\mathrm{m}},(\mathrm{m}+5) / 2\right)=\mathrm{C}_{\mathrm{n}}\left(\mathrm{u}_{2 \mathrm{~m}+2-\mathrm{i}}, \mathrm{PC}_{\mathrm{m}},(\mathrm{m}+5) / 2\right), \mathrm{i}=1,2, \ldots, \mathrm{m}-1$

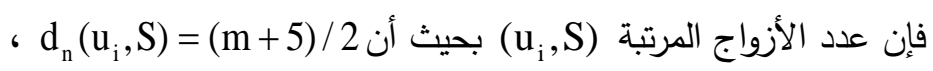
لكل

$2 \sum_{\mathrm{i}=1}^{\mathrm{m}-1}\left[\left(\begin{array}{c}\mathrm{p}-3(\mathrm{~m}-1) / 2-3\lceil\mathrm{i} / 2\rceil \\ \mathrm{n}-1\end{array}\right)-\left(\begin{array}{c}\mathrm{p}-3(\mathrm{~m}+1) / 2-3\lceil\mathrm{i} / 2\rceil \\ \mathrm{n}-1\end{array}\right)\right]$

$=4\left(\begin{array}{c}\mathrm{p}-3(\mathrm{~m}+1) / 2 \\ \mathrm{n}-1\end{array}\right)-4\left(\begin{array}{c}2 \\ \mathrm{n}-1\end{array}\right)$.

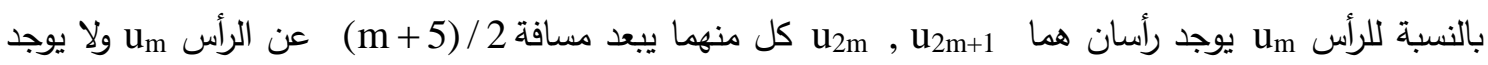

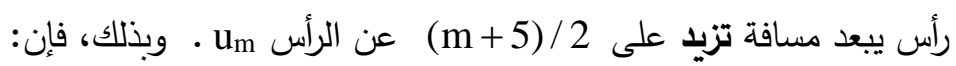

$\mathrm{C}_{\mathrm{n}}\left(\mathrm{u}_{\mathrm{m}}, \mathrm{PC}_{\mathrm{m}},(\mathrm{m}+5) / 2\right)=\left(\begin{array}{c}2 \\ \mathrm{n}-1\end{array}\right)$.

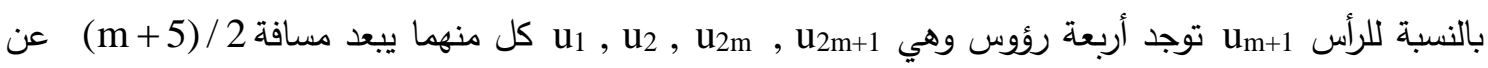

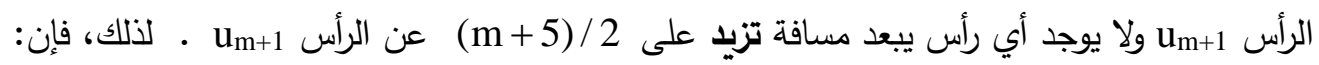
$\mathrm{C}_{\mathrm{n}}\left(\mathrm{u}_{\mathrm{m}+1}, \mathrm{PC}_{\mathrm{m}},(\mathrm{m}+5) / 2\right)=\left(\begin{array}{c}4 \\ \mathrm{n}-1\end{array}\right)$.

واضح أن:

$\mathrm{C}_{\mathrm{n}}\left(\mathrm{u}_{\mathrm{m}}, \mathrm{PC}_{\mathrm{m}},(\mathrm{m}+5) / 2\right)=\mathrm{C}_{\mathrm{n}}\left(\mathrm{u}_{\mathrm{m}+2}, \mathrm{PC}_{\mathrm{m}},(\mathrm{m}+5) / 2\right)$ 


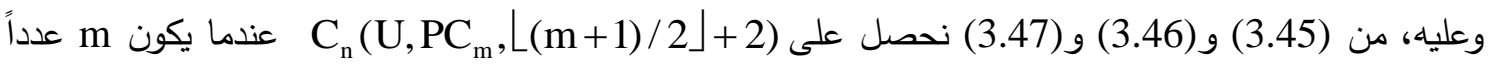

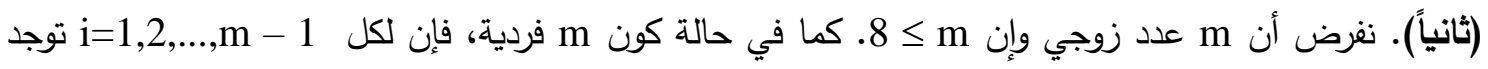
ثلاثة رؤوس وهي

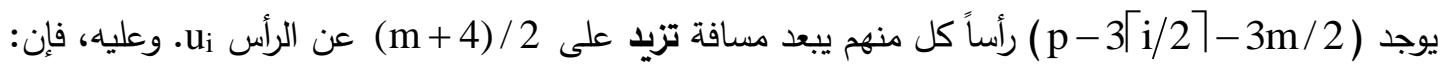
$\mathrm{C}_{\mathrm{n}}\left(\mathrm{u}_{\mathrm{i}}, \mathrm{PC}_{\mathrm{m}},(\mathrm{m}+4) / 2\right)=\left(\begin{array}{c}\mathrm{p}-3\lceil\mathrm{i} / 2\rceil-3 \mathrm{~m} / 2+3 \\ \mathrm{n}-1\end{array}\right)-\left(\begin{array}{c}\mathrm{p}-3\lceil\mathrm{i} / 2\rceil-3 \mathrm{~m} / 2 \\ \mathrm{n}-1\end{array}\right)$

$\mathrm{C}_{\mathrm{n}}\left(\mathrm{u}_{\mathrm{i}}, \mathrm{PC}_{\mathrm{m}},(\mathrm{m}+4) / 2\right)=\mathrm{C}_{\mathrm{n}}\left(\mathrm{u}_{2 \mathrm{~m}+2-\mathrm{i}}, \mathrm{PC}_{\mathrm{m}},(\mathrm{m}+4) / 2\right)$, ولما كان

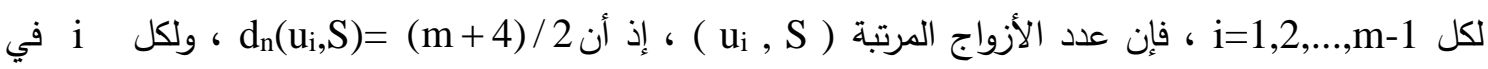
هو $\{1,2, \ldots, \mathrm{m}-1 ; \mathrm{m}+3, \mathrm{~m}+4, \ldots, 2 \mathrm{~m}+1\}$ $2 \sum_{\mathrm{i}=1}^{\mathrm{m}-1}\left[\left(\begin{array}{c}\mathrm{p}+3-3\lceil\mathrm{i} / 2\rceil-3 \mathrm{~m} / 2 \\ \mathrm{n}-1\end{array}\right)-\left(\begin{array}{c}\mathrm{p}-3 \mathrm{~m} / 2-3\lceil\mathrm{i} / 2\rceil \\ \mathrm{n}-1\end{array}\right)\right]$

$$
=4\left(\begin{array}{c}
\mathrm{p}-\frac{3}{2} \mathrm{~m} \\
\mathrm{n}-1
\end{array}\right)-2\left(\begin{array}{c}
5 \\
\mathrm{n}-1
\end{array}\right)-2\left(\begin{array}{c}
2 \\
\mathrm{n}-1
\end{array}\right) \text {. }
$$

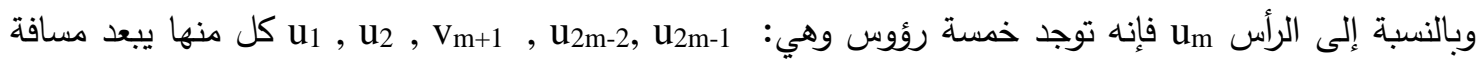

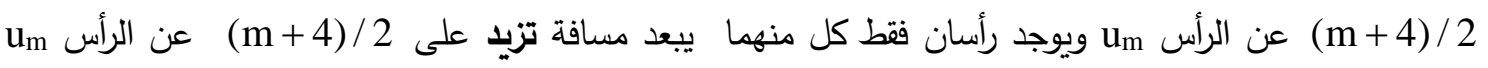
وعليه ، فأن $\mathrm{C}_{\mathrm{n}}\left(\mathrm{u}_{\mathrm{m}}, \mathrm{PC}_{\mathrm{m}},(\mathrm{m}+4) / 2\right)=\left(\begin{array}{c}7 \\ \mathrm{n}-1\end{array}\right)-\left(\begin{array}{c}2 \\ \mathrm{n}-1\end{array}\right)$.

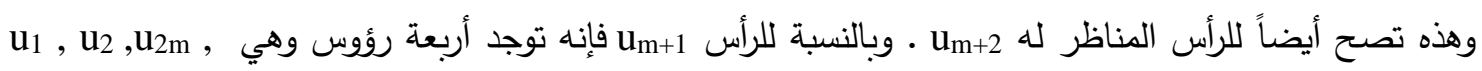

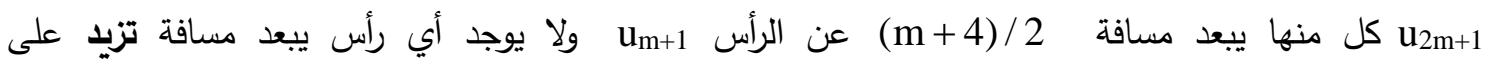
$\mathrm{C}_{\mathrm{n}}\left(\mathrm{u}_{\mathrm{m}+1}, \mathrm{PC}_{\mathrm{m}},(\mathrm{m}+4) / 2\right)=\left(\begin{array}{c}4 \\ \mathrm{n}-1\end{array}\right)$.

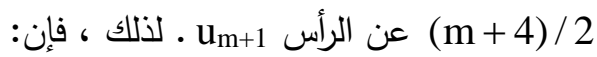

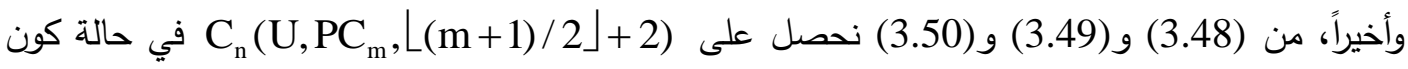

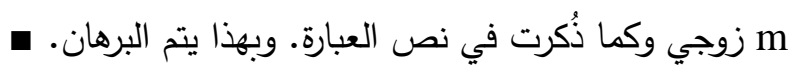
العبارة 3.11 : إذا كان $\mathrm{C}_{\mathrm{n}}\left(\mathrm{U}, \mathrm{PC}_{\mathrm{m}}, \mathrm{k}\right)=4\left(\begin{array}{c}\mathrm{p}+6-3 \mathrm{k} \\ \mathrm{n}-1\end{array}\right)$.

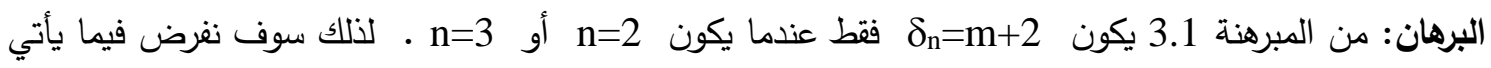
أن ثلاثة رؤوس وهي 


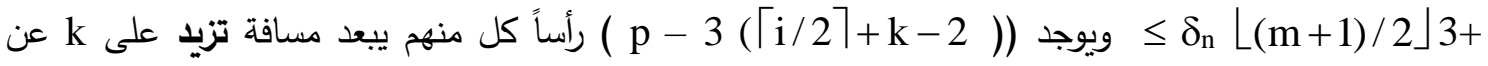

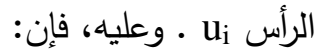

$\mathrm{C}_{\mathrm{n}}\left(\mathrm{u}_{\mathrm{i}}, \mathrm{PC}_{\mathrm{m}}, \mathrm{k}\right)=\left(\begin{array}{c}\mathrm{p}-3(\lceil\mathrm{i} / 2\rceil+\mathrm{k}-3) \\ \mathrm{n}-1\end{array}\right)-\left(\begin{array}{c}\mathrm{p}-3(\lceil\mathrm{i} / 2\rceil+\mathrm{k}-2) \\ \mathrm{n}-1\end{array}\right), \mathrm{i}=1,2, \ldots, 2(\mathrm{~m}-\mathrm{k}+2)$

فضلاً عن ذلك يوجد رأسان فقط هما un+1, u2m كل منهما يبعد مسافة k

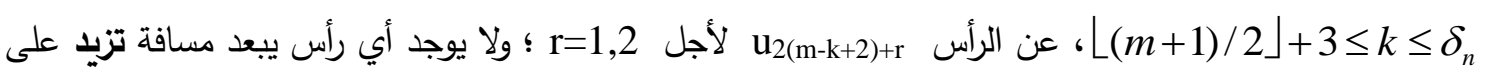
k عن هذا الرأس. لذلك : k

$\mathrm{C}_{\mathrm{n}}\left(\mathrm{u}_{2(\mathrm{~m}-\mathrm{k}+2)+\mathrm{r}}, \mathrm{PC}_{\mathrm{m}}, \mathrm{k}\right)=\left(\begin{array}{c}2 \\ \mathrm{n}-1\end{array}\right), \quad \mathrm{r}=1,2$

واضح أن:

$\mathrm{C}_{\mathrm{n}}\left(\mathrm{u}_{\mathrm{i}}, \mathrm{PC}_{\mathrm{m}}, \mathrm{k}\right)=\mathrm{C}_{\mathrm{n}}\left(\mathrm{u}_{2 \mathrm{~m}-\mathrm{i}+2}, \mathrm{PC}_{\mathrm{m}}, \mathrm{k}\right), \quad \mathrm{i}=1,2, \ldots, 2(\mathrm{~m}-\mathrm{k}+3)$

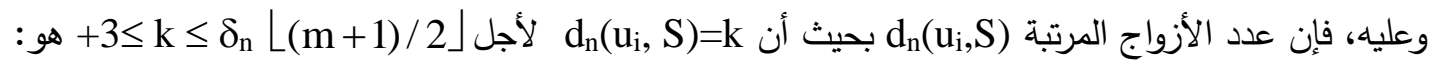

$2 \sum_{\mathrm{i}=1}^{2(\mathrm{~m}-\mathrm{k}+2)}\left[\left(\begin{array}{c}\mathrm{p}-3(\lceil\mathrm{i} / 2\rceil+\mathrm{k}-3) \\ \mathrm{n}-1\end{array}\right)-\left(\begin{array}{c}\mathrm{p}-3(\lceil\mathrm{i} / 2\rceil+\mathrm{k}-2) \\ \mathrm{n}-1\end{array}\right)\right]+4\left(\begin{array}{c}2 \\ \mathrm{n}-1\end{array}\right)$

$=4\left(\begin{array}{c}\mathrm{p}-3 \mathrm{k}+6 \\ \mathrm{n}-1\end{array}\right)-4\left(\begin{array}{c}2 \\ \mathrm{n}-1\end{array}\right)+4\left(\begin{array}{c}2 \\ \mathrm{n}-1\end{array}\right)=4\left(\begin{array}{c}\mathrm{p}-3 \mathrm{k}+6 \\ \mathrm{n}-1\end{array}\right)$.

عندما يكون n=m+2 فn=3 فإن الصيغة أعلاه تساوي 4 وهذه تساوي بالضبط عدد الأزواج المرتبة بمسافة

m+2

$\left(\mathrm{u}_{1},\left\{\mathrm{u}_{2 \mathrm{~m}}, \mathrm{u}_{2 \mathrm{~m}+1}\right\}\right),\left(\mathrm{u}_{2},\left\{\mathrm{u}_{2 \mathrm{~m}}, \mathrm{u}_{2 \mathrm{~m}+1}\right\}\right),\left(\mathrm{u}_{2 \mathrm{~m}+1},\left\{\mathrm{u}_{1}, \mathrm{u}_{2}\right\}\right),\left(\mathrm{u}_{2 \mathrm{~m}},\left\{\mathrm{u}_{1}, \mathrm{u}_{2}\right\}\right)$

ولا يوجد غيرها على مسافة تساوي m+2 ـ ولذلك فإن الصيغة (3.51) صحيحة لهذه القيم لـ k وبهذا يتم البرهان.

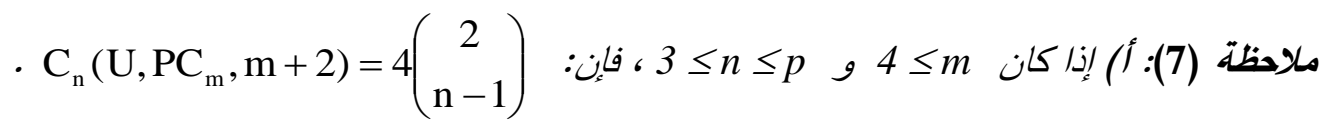

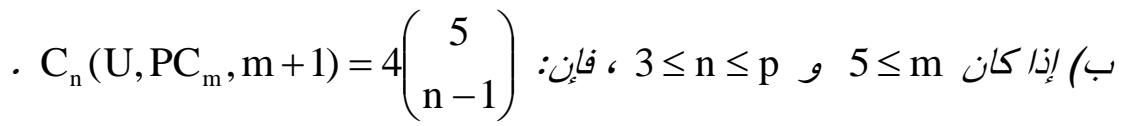

ج)

وأخيراً، نستخلص زبدة هذا البحث وهي المبرهنة الآتية والتي تتتج من كافة العبارات والملاحظات السابقة.

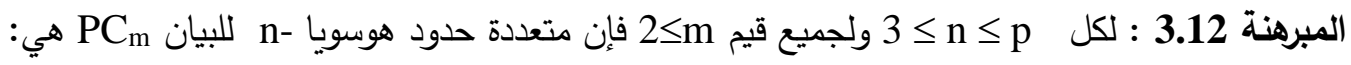

$\mathrm{H}_{\mathrm{n}}\left(\mathrm{PC}_{\mathrm{m}} ; \mathrm{x}\right)=\mathrm{p}\left(\begin{array}{l}\mathrm{p}-1 \\ \mathrm{n}-2\end{array}\right)+\left[\mathrm{p}\left(\begin{array}{l}\mathrm{p}-1 \\ \mathrm{n}-1\end{array}\right)-2(\mathrm{~m}-1)\left(\begin{array}{l}\mathrm{p}-4 \\ \mathrm{n}-1\end{array}\right)-(\mathrm{m}+4)\left(\begin{array}{l}\mathrm{p}-3 \\ \mathrm{n}-1\end{array}\right)\right] \mathrm{x}+\sum_{\mathrm{k}=2}^{\delta_{\mathrm{n}}} \mathrm{C}_{\mathrm{n}}\left(\mathrm{PC}_{\mathrm{m}}, \mathrm{k}\right) \mathrm{x}^{\mathrm{k}}$

إذ أن $\mathrm{C}_{\mathrm{n}}\left(\mathrm{PC}_{\mathrm{m}}, \mathrm{k}\right)=\mathrm{C}_{\mathrm{n}}\left(\mathrm{V}, \mathrm{PC}_{\mathrm{m}}, \mathrm{k}\right)+\mathrm{C}_{\mathrm{n}}\left(\mathrm{U}, \mathrm{PC}_{\mathrm{m}}, \mathrm{k}\right)$ 
والتي تم تحديدها في العبارات من 3.1 إلى 3.11 والملاحظات من (1) إلى (7) . نتيجة 3.13 : متعددة حدود هوسويا للبيان PC ، PC 2 هي : $\mathrm{H}\left(\mathrm{PC}_{\mathrm{m}} ; \mathrm{x}\right)=(3 \mathrm{~m}+2)+(4 \mathrm{~m}+1) \mathrm{x}+\sum_{\mathrm{k}=2}^{\mathrm{m}+2} \mathrm{C}\left(\mathrm{PC}_{\mathrm{m}}, \mathrm{k}\right) \mathrm{x}^{\mathrm{k}}$,

$$
R_{k}= \begin{cases}4 m-3 k+5 & , 2 \leq k \leq 4 \\ 5 m-12 & , k=5 \\ 6(m-k)+16 & , 6 \leq k \leq m+1 \\ 5 & , k=m+2\end{cases}
$$

$\mathrm{C}\left(\mathrm{PC}_{\mathrm{m}}, \mathrm{k}\right)=3(\mathrm{~m}-\mathrm{k})+5+\mathrm{R}_{\mathrm{k}}$ إذ أن

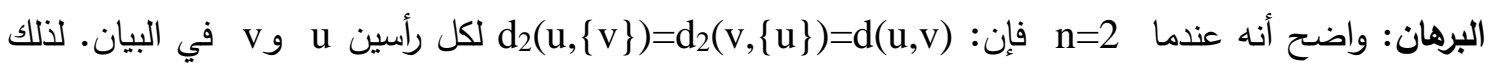

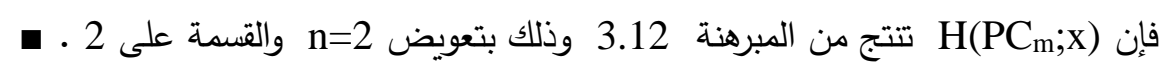
نتيجة 3.14 : دليل وينر للبيان PC ، إذ أن m 2 إن 2 هو: $\mathrm{W}\left(\mathrm{PC}_{\mathrm{m}}\right)=\frac{1}{2}\left(3 \mathrm{~m}^{3}+21 \mathrm{~m}^{2}-6 \mathrm{~m}+14\right)$.

البرهان: نأخذ مشتقة H(PC $\mathrm{W}\left(\mathrm{PC}_{\mathrm{m}}\right)=4 \mathrm{~m}+1+\sum_{\mathrm{k}=2}^{\mathrm{m}+2} \mathrm{k}\left[3(\mathrm{~m}-\mathrm{k})+5+\mathrm{R}_{\mathrm{k}}\right]$

$$
\begin{aligned}
& =4 \mathrm{~m}+1+2[3 \mathrm{~m}-6+5+(4 \mathrm{~m}-1)]+3[3 \mathrm{~m}-9+5+(4 \mathrm{~m}-9+5)] \\
& +4[3 \mathrm{~m}-12+5+(4 \mathrm{~m}-12+5)]+5[3 \mathrm{~m}-15+5+(5 \mathrm{~m}-12)] \\
& +\sum_{\mathrm{k}=6}^{\mathrm{m}+1} \mathrm{k}[3 \mathrm{~m}-3 \mathrm{k}+5+(6 \mathrm{~m}-6 \mathrm{k}+16)]+(\mathrm{m}+2)[3 \mathrm{~m}-3(\mathrm{~m}+2)+5+(5)] \\
& =111 \mathrm{~m}-185+\sum_{\mathrm{k}=6}^{\mathrm{m}+1} \mathrm{k}[9 \mathrm{~m}+21-9 \mathrm{k}]=\frac{1}{2}\left(3 \mathrm{~m}^{3}+21 \mathrm{~m}^{2}-6 \mathrm{~m}+14\right) .
\end{aligned}
$$




$$
\begin{aligned}
& \text { المصادر } \\
& \text { الدباغ، أحمد محمد علي (2005)، "متعددات حدود وينر لتعيم المسافة في البيانات"، رسالة ماجستير ، } \\
& \text { غير منشورة، جامعة الموصل، العراق. } \\
& \text { علي، علي عزيز (1983)، مقدمة في نظرية البيان، ط 1، الموصل: مؤسسة دار الكتب بجامعة } \\
& \text { الموصل. } \\
& \text { علي، علي عزيز، علي ،أحدد محمد (2006)، متعددات حدود وينر لتعميم المسافة لبعض البيانات }
\end{aligned}
$$

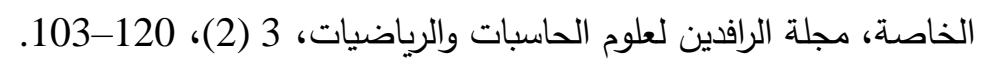

[4] Ahmed, H. G.; (2007). On Wiener Polynomials of n-Distance in Graphs, M.Sc. Thesis, unpublished, Dohuk University, Dohuk, Iraq.

[5] Buckley, F. and Harary, F.; (1990). Distance in Graphs. New York: AddisonWesley.

[6] Chartrand, G. and Lesniak, L.; (1986). Graphs and Digraphs, $2^{\text {nd }}$ ed., California :Wadsworth Inc.

[7] Dankelman, P., Goddard, W., Henning, M.A. and Swart, H.C.; (1999). "Generalized eccentricity, radius, and diameter in graphs", Foundation for Research Development. c 1999 John Wiely \& Sons, Inc. Networks 34, 312-319. 